1 Hacettepe Journal of Mathematics and Statistics

$\bigcap$ Volume 48 (4) (2019), 1250-1276

ResearCh ARTICLE

\title{
Restricted Liu estimator in generalized linear models: Monte Carlo simulation studies on gamma and Poisson distributed responses
}

\author{
Fikriye Kurtoğlu*† (1) and M. Revan Özkale ${ }^{\ddagger}$ (1)
}

\begin{abstract}
In this study, we introduce iterative restricted Liu estimator to combat multicollinearity in generalized linear models. We also obtain necessary and sufficient conditions for the superiority of the first-order approximated restricted Liu estimator over the first-order approximated maximum likelihood and Liu estimators by the approximated mean squared error criterion. The results are illustrated by conducting simulation studies and numerical examples.
\end{abstract}

Keywords: Restricted Liu estimation, Multicollinearity, Generalized linear models, Mean squared error, Gamma distribution, Poisson distribution.

Mathematics Subject Classification (2010): 62J12, 62J07

Received : 06.04.2018 Accepted: 19.09.2018 Doi : 10.15672/HJMS.2018.624

\section{Introduction}

Collinearity has long been recognised as a potential source of problem in the estimation, computation and interpretation of linear model parameters [1]. Although maximum likelihood (ML) estimator is the most widely used method in linear regression, ML estimates become unstable and covariance matrix of the ML estimator inflates when collinearity exists among the explanatory variables. Therefore, in the existence of the multicollinearity, estimators such as restricted estimator, Liu estimator [9], the restricted Liu estimator [7] and others were proposed as alternative to the ML estimator to reduce the adverse effects of the multicollinearity. As in the case in linear regression, collinearity causes difficulty in the interpretation of estimated regression coefficients in generalized

\footnotetext{
*Çukurova University, Faculty of Science and Letters, Department of Statistics, Adana, 01330, Turkey, Email: fkurtoglu@cu.edu.tr

${ }^{\dagger}$ Corresponding Author.

${ }^{\ddagger}$ Çukurova University, Faculty of Science and Letters, Department of Statistics, Adana, 01330, Turkey, Email: mrevan@cu.edu.tr
} 
linear models (GLMs). Mackinnon and Puterman [10] study how to detect the collinearity in GLMs. Marx and Smith [12] present a principle component estimator for GLMs, and show that it can be useful with the presence of an ill-conditioned information matrix. Nyquist [15] considers the maximum likelihood estimation in GLMs under linear restrictions on the parameters. Segerstedt [17] introduces the ordinary ridge regression estimator for GLMs. Kurtoğlu and Özkale [8] derive Liu estimator for GLMs. In addition to observations on the response and explanatory variables, auxiliary information on the vector of regression coefficients can exist. Then, auxiliary information, especially exact linear restrictions, can be used to overcome multicollinearity. Therefore, it is the objective of this paper to present a restricted Liu estimator in GLMs. Both iterative and first-order approximated restricted Liu (FOARL) estimators are developed using quadratic penalized likelihood. Because there are different estimators used in the case of multicollinearity in linear regression, these estimators can also be defined for GLMs. As in linear regression, estimation in GLMs is also sensitive to multicollinearity.

This article is organized as follows. In Section 2, we propose iterative restricted Liu estimator in GLMs in the existence of multicollinearity. In Section 3, the mean squared error (MSE) properties of FOARL estimator in GLMs and the performance of the FOARL estimator are obtained, numerical examples are given in Section 4. Finally, two Monte Carlo simulation studies are presented in Section 5. In Section 6, the conclusions of the paper are presented.

\section{The FOARL Estimation in GLMs}

Suppose that $y_{1}, \ldots, y_{n}$ be the observations of independent random variables $Y_{1}, \ldots, Y_{n}$, each of which has the probability density function such that the mean of $Y_{i}$ is $\mu_{i}$ and the corresponding canonical parameter is $\theta_{i}=g\left(\mu_{i}\right), i=1, \ldots, n$. The exponential family can be written in the form

$$
f\left(y_{i}, \theta_{i}, \phi\right)=\exp \left[\frac{\theta_{i} y_{i}-b\left(\theta_{i}\right)}{a(\phi)}+c\left(y_{i}, \phi\right)\right], \quad i=1, \ldots, n
$$

where $a(\cdot), b(\cdot)$ and $c(\cdot)$ are known functions and $\phi>0$ is a dispersion parameter, which in some families takes on a fixed known value, while in other families it is an unknown parameter to be estimated from the data along with $\theta$. For each observation values of a set of $q$ explanatory variables, $x_{i}^{\top}=\left(x_{i 1}, \ldots, x_{i q}\right)$ is also recorded where $x_{i}^{\top}$ is the $i$ th row of the design matrix $\mathbf{X}=\left(x_{1}, \ldots, x_{n}\right)^{\top}$ which denotes the $n \times q$ matrix of explanatory variables, $\eta_{i}=x_{i}^{\top} \boldsymbol{\beta}$ is the linear predictor where $\boldsymbol{\beta}=\left(\beta_{1}, \ldots, \beta_{q}\right)^{\top}$ is the $q \times 1$ vector of parameters, also known as regression coefficients. The log-likelihood function is given by

$$
l(\theta, \phi, y)=\sum_{i=1}^{n}\left[\frac{\theta_{i} y_{i}-b\left(\theta_{i}\right)}{a(\phi)}+c\left(y_{i}, \phi\right)\right]
$$

where $\boldsymbol{\theta}=\left(\theta_{1}, \ldots, \theta_{n}\right)^{\top}, \mathbf{y}=\left(y_{1}, \ldots, y_{n}\right)^{\top}$.

We suppose that in addition to the linear predictor, the set of $q$ linearly independent restrictions on the parameter vector $\boldsymbol{\beta}$ exist: $\mathbf{R}_{t}^{\top} \boldsymbol{\beta}=\mathbf{r}_{t}, t=1, \ldots, T$, where $\mathbf{R}_{t}^{\top}=$ $\left(R_{t 1}, \ldots, R_{t q}\right)$ are $q \times 1$ vectors and $r_{t}$ are scalars. Alternatively, we define the restricted Liu estimator for GLMs with the log-likelihood function in (2.1) with respect to quadratic 
penaly function $\left(\mathbf{R}_{t}^{\top} \boldsymbol{\beta}-r_{t}\right)^{2}$. The objective function is

$$
\begin{aligned}
\psi(\boldsymbol{\beta}, d, \boldsymbol{\lambda})= & {\left[\sum_{i=1}^{n} \frac{\theta_{i} y_{i}-b\left(\theta_{i}\right)}{a(\phi)}+c\left(y_{i}, \phi\right)\right] } \\
& -\frac{1}{2 a(\phi)}(\boldsymbol{\beta}-d \hat{\boldsymbol{\beta}})^{\top}(\boldsymbol{\beta}-d \hat{\boldsymbol{\beta}})-\frac{1}{2 a(\phi)} \sum_{t=1}^{T} \lambda_{t}\left(\mathbf{R}_{t}^{\top} \boldsymbol{\beta}-\mathbf{r}_{t}\right)^{2},
\end{aligned}
$$

where $0<d<1$ is Liu-biasing parameter, Lagrange multipliers and $\hat{\boldsymbol{\beta}}$ is the iteratively reweighted least squares (IRLS) estimator evaluated at the final iteration.

Differentiating $\psi(\boldsymbol{\beta}, d, \boldsymbol{\lambda})$ with respect to $\boldsymbol{\beta}$ and making use of Fisher's method of scoring, we get the $q \times 1$ vector

$$
\begin{aligned}
& \left.Q(\boldsymbol{\beta}, d, \boldsymbol{\lambda})\right|_{\beta=\hat{\beta}_{r}(d)^{(m)}}=\left[\frac{\partial \psi(\boldsymbol{\beta}, d, \boldsymbol{\lambda})}{\partial \boldsymbol{\beta}}\right]_{\beta=\hat{\beta}_{r}(d)}{ }^{(m)} \\
& =\frac{1}{a(\phi)}\left[\mathbf{X}^{\top} \mathbf{W D}(\mathbf{y}-\boldsymbol{\mu})-\boldsymbol{\beta}+d \hat{\boldsymbol{\beta}}-\mathbf{R}^{\top} \boldsymbol{\Lambda}^{*}(\mathbf{R} \boldsymbol{\beta}-\mathbf{r})\right]_{\beta=\hat{\beta}_{r}(d)^{(m)}},
\end{aligned}
$$

where $\boldsymbol{\Lambda}^{*}=\operatorname{diag}\left(\lambda_{1}, \ldots, \lambda_{T}\right)$ is the matrix of Lagrange multipliers, $\mathbf{R}^{\top}=\left(R_{1} \ldots R_{T}\right)$ is the $q \times T$ known auxiliary information matrix that expresses the structure of information on the individual parameters or some linear combinatory of these parameters, $\mathbf{r}=\left(r_{1} \ldots r_{T}\right)^{\top}$ is the $1 \times T$ vector of scalars and $\mathbf{W}=\operatorname{diag}\left\{w_{i i}\right\}$ is the $n \times n$ diagonal matrix with weights $w_{i i}=\left\{1 / \operatorname{var}\left(Y_{i}\right)\right\}\left(d \mu_{i} / d \eta_{i}\right)^{2}$. Both $R$ and $r$ are assumed to be known and in addition $\operatorname{rank}(R)=T$ is assumed. The $j$ th $(j=1, \ldots, q)$ element of $Q(\boldsymbol{\beta}, d, \boldsymbol{\lambda})$ is

$$
\begin{aligned}
& q_{j}(\boldsymbol{\beta}, d, \boldsymbol{\lambda})=\frac{\partial \psi(\boldsymbol{\beta}, d, \boldsymbol{\lambda})}{\partial \beta_{j}} \\
& =\frac{1}{a(\phi)}\left[\sum_{i=1}^{n} \frac{y_{i}-\mu_{i}}{w_{i}} g^{\prime}\left(\mu_{i}\right) x_{i j}-\beta_{j}+d \hat{\beta}_{j}-\sum_{t=1}^{T} \mathbf{R}_{t j} \lambda_{t}\left(\mathbf{R}_{t}^{\top} \boldsymbol{\beta}-r_{t}\right)\right] .
\end{aligned}
$$

Second-order partial derivatives of $\psi(\boldsymbol{\beta}, d, \boldsymbol{\lambda})$ gives the Hessian matrix $\mathrm{H}_{l}(\boldsymbol{\beta}, d, \boldsymbol{\lambda})=$ $\partial^{2} \psi(\boldsymbol{\beta}, d, \boldsymbol{\lambda}) / \partial \boldsymbol{\beta} \partial \boldsymbol{\beta}^{\prime}$ having the $(j, v)$ th entry as

$$
\begin{aligned}
\frac{\partial^{2} \psi(\boldsymbol{\beta}, d, \boldsymbol{\lambda})}{\partial \beta_{j} \partial \beta_{v}}= & \frac{1}{a(\phi)}\left[\sum_{i=1}^{n}\left(y_{i}-\mu_{i}\right) \frac{\partial}{\partial \beta_{v}}\left(\frac{g^{\prime}\left(\mu_{i}\right)}{w_{i}} x_{i j}\right)\right. \\
& \left.-\sum_{i=1}^{n} \frac{x_{i j}}{w_{i}} x_{i v}-\delta_{j v}-\sum_{t=1}^{T} \lambda_{t} \mathbf{R}_{t j} \mathbf{R}_{t v}\right],
\end{aligned}
$$

where $\delta_{j v}=1$ if $j=v$ and zero otherwise.

Taking the expected value of both sides of (2.3), we get

$$
\begin{aligned}
s_{j v}(\boldsymbol{\beta}, d, \boldsymbol{\lambda}) & =-E\left[\frac{\partial^{2} \psi(\boldsymbol{\beta}, d, \boldsymbol{\lambda})}{\partial \beta_{j} \partial \beta_{v}}\right] \\
& =\frac{1}{a(\phi)}\left[\sum_{i=1}^{n} \frac{1}{w_{i}} x_{i j} x_{i v}+\delta_{j v}+\sum_{t=1}^{T} \lambda_{t} \mathbf{R}_{t j} \mathbf{R}_{t v}\right] .
\end{aligned}
$$

Consequently, the expected value of $\mathrm{H}_{l}(\boldsymbol{\beta}, d, \boldsymbol{\lambda})$ is of the form

$$
\mathrm{E}\left[\mathrm{H}_{l}(\boldsymbol{\beta}, d, \boldsymbol{\lambda})\right]=\frac{1}{a(\phi)}\left[\left(\mathbf{X}^{\top} \mathbf{W X}+\mathbf{I}\right)+\mathbf{R}^{\top} \boldsymbol{\Lambda}^{*} \mathbf{R}\right]
$$

Application of the Fisher scoring method yields 


$$
\begin{aligned}
\hat{\boldsymbol{\beta}}_{r}(d)^{(m+1)}=\hat{\boldsymbol{\beta}}_{r}(d)^{(m)}+\left\{\left\{\mathrm{E}\left[\mathrm{H}_{l}(\boldsymbol{\beta}, d, \boldsymbol{\lambda})\right]\right\}^{-1}\left[\frac{\partial \psi(\boldsymbol{\beta}, d, \boldsymbol{\lambda})}{\partial \boldsymbol{\beta}}\right]\right\}_{\beta=\hat{\beta}_{r}(d)}^{(m)} \\
=\hat{\boldsymbol{\beta}}(d)^{(m+1)}-\left(\mathbf{X}^{\top} \mathbf{W}^{(m)} \mathbf{X}+\mathbf{I}\right)^{-1} \mathbf{R}^{\top}\left[\left(\boldsymbol{\Lambda}^{*}\right)^{-1}\right. \\
+ \\
\left.+\mathbf{R}\left(\mathbf{X}^{\top} \mathbf{W}^{(m)} \mathbf{X}+\mathbf{I}\right)^{-1} \mathbf{R}^{\top}\right]^{-1}\left(\mathbf{R} \hat{\boldsymbol{\beta}}(d)^{(m+1)}-\mathbf{r}\right),
\end{aligned}
$$

where $\hat{\boldsymbol{\beta}}(d)^{(m+1)}=\left(\mathbf{X}^{\top} \mathbf{W}^{(m)} \mathbf{X}+\mathbf{I}\right)^{-1}\left(\mathbf{X}^{\top} \mathbf{W}^{(m)} \mathbf{X}+d \mathbf{I}\right) \hat{\boldsymbol{\beta}}^{(m+1)}$ is in the form of the proposed Liu estimator by Kurtoğlu and Özkale [8] and $\hat{\boldsymbol{\beta}}^{(m+1)}$ is in the form of the ML estimator in GLMs and both $\hat{\boldsymbol{\beta}}(d)^{(m+1)}$ and $\hat{\boldsymbol{\beta}}^{(m+1)}$ are evaluated at the iteration algorithm (derivation details are given in Appendix A).

The $(m+1)$ th approximation of the restricted Liu estimator is finally obtained as

$$
\begin{aligned}
\hat{\boldsymbol{\beta}}_{r}(d)^{(m+1)}= & \lim _{\lambda_{1}, \ldots, \lambda_{q} \rightarrow \infty} \hat{\boldsymbol{\beta}}_{r}(d)^{(m+1)} \\
= & \hat{\boldsymbol{\beta}}(d)^{(m+1)}-\left(\mathbf{X}^{\top} \mathbf{W}^{(m)} \mathbf{X}+\mathbf{I}\right)^{-1} \mathbf{R}^{\top}\left[\mathbf{R}\left(\mathbf{X}^{\top} \mathbf{W}^{(m)} \mathbf{X}+\mathbf{I}\right)^{-1} \mathbf{R}^{\top}\right]^{-1} \\
& \times\left(\mathbf{R} \hat{\boldsymbol{\beta}}(d)^{(m+1)}-\mathbf{r}\right) .
\end{aligned}
$$

In the special case of $d=0, \hat{\boldsymbol{\beta}}_{r}(d)^{(m+1)}$ equals to the restricted estimator which is introduced by Nyquist [15]. The estimator in (2.5) is calculated iteratively. The estimator in the first step can be written as

$$
\begin{aligned}
\hat{\boldsymbol{\beta}}_{r}(d)^{(1)}= & \hat{\boldsymbol{\beta}}(d)^{(1)}-\left(\mathbf{X}^{\top} \mathbf{W}^{(0)} \mathbf{X}+\mathbf{I}\right)^{-1} \mathbf{R}^{\top}\left[\mathbf{R}\left(\mathbf{X}^{\top} \mathbf{W}^{(0)} \mathbf{X}+\mathbf{I}\right)^{-1} \mathbf{R}^{\top}\right]^{-1} \\
& \times\left(\mathbf{R} \hat{\boldsymbol{\beta}}(d)^{(1)}-\mathbf{r}\right),
\end{aligned}
$$

where $\hat{\boldsymbol{\beta}}(d)^{(1)}=\left(\mathbf{X}^{\top} \mathbf{W}^{(0)} \mathbf{X}+\mathbf{I}\right)^{-1}\left(\mathbf{X}^{\top} \mathbf{W}^{(0)} \mathbf{X}+d \mathbf{I}\right) \hat{\boldsymbol{\beta}}^{(1)}$ is the first-order approximated Liu (FOAL) estimator, $\hat{\boldsymbol{\beta}}^{(1)}=\left(\mathbf{X}^{\top} \mathbf{W}^{(0)} \mathbf{X}\right)^{-1} \mathbf{X}^{\top} \mathbf{W}^{(0)} \mathbf{z}^{(0)}$ is the first-order approximated ML (FOAML) estimator and $\mathbf{z}^{(0)}=\mathbf{X} \boldsymbol{\beta}^{(0)}+[\mathbf{D}(\mathbf{y}-\boldsymbol{\mu})]_{\beta=\beta^{(0)}}$ is the working response. The first-order approximated restricted ML (FOARML) estimator in GLMs given by Nyquist [15] is

$$
\hat{\boldsymbol{\beta}}_{r}^{(1)}=\hat{\boldsymbol{\beta}}^{(1)}-\left(\mathbf{X}^{\top} \mathbf{W}^{(0)} \mathbf{X}\right)^{-1} \mathbf{R}^{\top}\left[\mathbf{R}\left(\mathbf{X}^{\top} \mathbf{W}^{(0)} \mathbf{X}\right)^{-1} \mathbf{R}^{\top}\right]^{-1}\left(\mathbf{R} \hat{\boldsymbol{\beta}}^{(1)}-\mathbf{r}\right) .
$$

Thus, the weight matrix and the working response of $\hat{\boldsymbol{\beta}}_{r}(d)^{(1)}, \hat{\boldsymbol{\beta}}(d)^{(1)}, \hat{\boldsymbol{\beta}}^{(1)}$ and $\hat{\boldsymbol{\beta}}_{r}^{(1)}$ are calculated in the same $\boldsymbol{\beta}^{(0)}$ initial value or real parameter and all these four estimators have the same working response.

\section{Superiority of the FOARL estimator over other estimators by the matrix MSE criterion}

In this section, we compare the FOARL estimator to the FOAML, FOARML and FOAL estimators according to the matrix MSE criterion. We compute approximated biasing vector and variance covariance matrix of the estimator $\hat{\boldsymbol{\beta}}_{r}(d)^{(1)}$ as

$$
\begin{aligned}
& \operatorname{Bias}\left[\hat{\boldsymbol{\beta}}_{r}(d)^{(1)}\right]=(d-1) \mathbf{M}_{1} \boldsymbol{\beta}-\mathbf{S}_{1}^{-1} \mathbf{R}^{\top}\left(\mathbf{R} \mathbf{S}_{1}^{-1} \mathbf{R}^{\top}\right)^{-1}(\mathbf{R} \boldsymbol{\beta}-\mathbf{r}), \\
& \operatorname{Var}\left[\hat{\boldsymbol{\beta}}_{r}(d)^{(1)}\right]=a(\phi) \mathbf{M}_{1} \mathbf{S}_{d} \mathbf{S}_{1}^{-1} \mathbf{S}_{d} \mathbf{M}_{1},
\end{aligned}
$$

where $\mathbf{M}_{1}=\mathbf{S}_{1}^{-1}-\mathbf{S}_{1}^{-1} \mathbf{R}^{\top}\left(\mathbf{R S}_{1}^{-1} \mathbf{R}^{\top}\right)^{-1} \mathbf{R S}_{1}^{-1}, \mathbf{S}_{d}=\mathbf{X}^{\top} \mathbf{W}^{(0)} \mathbf{X}+d \mathbf{I}$, and $\mathbf{S}_{1}=\mathbf{X}^{\top} \mathbf{W}^{(0)} \mathbf{X}+$ I. Then, the matrix MSE of the estimator $\hat{\boldsymbol{\beta}}_{r}(d)^{(1)}$ is

$$
\begin{aligned}
\operatorname{MSE}\left[\hat{\boldsymbol{\beta}}_{r}(d)^{(1)}\right]= & a(\phi) \mathbf{M}_{1} \mathbf{S}_{d} \mathbf{S}^{-1} \mathbf{S}_{d} \mathbf{M}_{1}+\left[(d-1) \mathbf{M}_{1} \beta-\mathbf{S}_{1}^{-1} \mathbf{R}^{\top}\left(\mathbf{R S}_{1}^{-1} \mathbf{R}^{\top}\right)^{-1}\right. \\
& \times(\mathbf{R} \boldsymbol{\beta}-\mathbf{r})]\left[(d-1) \mathbf{M}_{1} \boldsymbol{\beta}-\mathbf{S}_{1}^{-1} \mathbf{R}^{\top}\left(\mathbf{R} \mathbf{S}_{1}^{-1} \mathbf{R}^{\top}\right)^{-1}(\mathbf{R} \boldsymbol{\beta}-\mathbf{r})\right]^{\top} .
\end{aligned}
$$

Similarly, the MSE matrices of the estimators $\hat{\boldsymbol{\beta}}^{(1)}, \hat{\boldsymbol{\beta}}_{r}^{(1)}$ and $\hat{\boldsymbol{\beta}}(d)^{(1)}$ are respectively as

$$
\operatorname{MSE}\left[\hat{\boldsymbol{\beta}}^{(1)}\right]=a(\phi) \mathbf{S}^{-1},
$$




$$
\begin{aligned}
\operatorname{MSE}\left[\hat{\boldsymbol{\beta}}_{r}^{(1)}\right]= & a(\phi) \mathbf{M}_{0}+\mathbf{S}^{-1} \mathbf{R}^{\top}\left(\mathbf{R} \mathbf{S}^{-1} \mathbf{R}^{\top}\right)^{-1} \\
& \times(\mathbf{R} \boldsymbol{\beta}-\mathbf{r})(\mathbf{R} \boldsymbol{\beta}-\mathbf{r})^{\top}\left[\mathbf{S}^{-1} \mathbf{R}^{\top}\left(\mathbf{R} \mathbf{S}^{-1} \mathbf{R}^{\top}\right)^{-1}\right]^{\top},
\end{aligned}
$$

and

$$
\operatorname{MSE}\left[\hat{\boldsymbol{\beta}}(d)^{(1)}\right]=a(\phi) \mathbf{S}_{1}^{-1} \mathbf{S}_{d} \mathbf{S}^{-1} \mathbf{S}_{d} \mathbf{S}_{1}^{-1}+(d-1)^{2} \mathbf{S}_{1}^{-1} \boldsymbol{\beta} \boldsymbol{\beta}^{\top} \mathbf{S}_{1}^{-1},
$$

where $\mathbf{S}=\mathbf{X}^{\top} \mathbf{W}^{(0)} \mathbf{X}$ and $\mathbf{M}_{0}=\mathbf{S}^{-1}-\mathbf{S}^{-1} \mathbf{R}^{\top}\left(\mathbf{R} \mathbf{S}^{-1} \mathbf{R}^{\top}\right)^{-1} \mathbf{R} \mathbf{S}^{-1}$.

3.1. Comparisons when the restrictions are true, i.e., $\boldsymbol{R} \boldsymbol{\beta}=\boldsymbol{r}$. We examine the matrix MSE comparisons of $\hat{\boldsymbol{\beta}}_{r}(d)^{(1)}$ and $\hat{\boldsymbol{\beta}}_{r}^{(1)}$ in GLMs when the restrictions hold true. We see that the MSE matrices of the estimators in GLMs are similar to that of the corresponding estimators in linear regression models. However, the points that should be emphasized are that the response in GLMs is from the exponential family while the response in linear regression is usually from normal distribution. Furthermore, the MSE matrices depend on the matrix $\mathbf{X}^{\top} \mathbf{W X}$ in GLMs while on the matrix $\mathbf{X}^{\top} \mathbf{X}$ in linear regression. As a consequence, although the results in their closed forms seem to be similar they are not exactly the same.

3.1.1. The comparison between the FOARL estimator and the FOAL estimator. For the superiority of $\hat{\boldsymbol{\beta}}_{r}(d)^{(1)}$ over $\hat{\boldsymbol{\beta}}(d)^{(1)}$ when $\mathbf{R} \boldsymbol{\beta}=\mathbf{r}$ holds true, Theorem 3.1 can be given. The difference $\boldsymbol{\Delta}_{1}=\operatorname{MSE}\left[\hat{\boldsymbol{\beta}}(d)^{(1)}\right]-\operatorname{MSE}\left[\hat{\boldsymbol{\beta}}_{r}(d)^{(1)}\right]$ is given by $\boldsymbol{\Delta}_{1}=\mathbf{B}-\mathbf{M}_{1} \mathbf{S}_{1} \mathbf{B} \mathbf{S}_{1} \mathbf{M}_{1}$ where $\mathbf{B}=\operatorname{MSE}\left[\hat{\boldsymbol{\beta}}(d)^{(1)}\right]$.

3.1. Theorem. The estimator $\hat{\boldsymbol{\beta}}_{r}(d)^{(1)}$ is superior to the estimator $\hat{\boldsymbol{\beta}}(d)^{(1)}$ by the criterion of matrix MSE if and only if $\lambda_{\max }\left(\mathbf{B}^{-1} \mathbf{M}_{1} \mathbf{S}_{1} \mathbf{B} \mathbf{S}_{1} \mathbf{M}_{1}\right) \leq 1$ where $\lambda_{\max }$ is the maximum eigenvalue of $\mathbf{M}_{1} \mathbf{S}_{1} \mathbf{B} \mathbf{S}_{1} \mathbf{M}_{1} \mathbf{B}^{-1}$.

Proof. For the superiority of $\hat{\boldsymbol{\beta}}_{r}(d)^{(1)}$ over $\hat{\boldsymbol{\beta}}(d)^{(1)}$, we examine the difference $\boldsymbol{\Delta}_{1}=$ $\operatorname{MSE}\left[\hat{\boldsymbol{\beta}}(d)^{(1)}\right]-\operatorname{MSE}\left[\hat{\boldsymbol{\beta}}_{r}(d)^{(1)}\right]$ is given by $\boldsymbol{\Delta}_{1}=\mathbf{B}-\mathbf{M}_{1} \mathbf{S}_{1} \mathbf{B S}_{1} \mathbf{M}_{1}$. By applying Theorem B.2 in Appendix B from Graybill [4] we can derive the necessary and sufficient condition for $\boldsymbol{\Delta}_{1}$ to be nonnegative definite (nnd). Since $B$ is positive definite (pd) and $\mathbf{M}_{1} \mathbf{S}_{1} \mathbf{B S}_{1} \mathbf{M}_{1}$ is a symmetric matrix, there exists a nonsingular matrix $\mathbf{Q}$ such that $\mathbf{Q}^{\top} \mathbf{B Q}=\mathbf{I}$ and $\mathbf{Q}^{\top} \mathbf{M}_{1} \mathbf{S}_{1} \mathbf{B S}_{1} \mathbf{M}_{1} \mathbf{Q}=\mathbf{\Lambda}$, where $\boldsymbol{\Lambda}$ is a diagonal elements are the roots of the polynomial equation $\left|\mathbf{M}_{1} \mathbf{S}_{1} \mathbf{B} \mathbf{S}_{1} \mathbf{M}_{1}-\lambda \mathbf{B}\right|=0$. Since

$$
\left|\mathbf{M}_{1} \mathbf{S}_{1} \mathbf{B S}_{1} \mathbf{M}_{1}-\lambda \mathbf{B}\right|=\left|\mathbf{M}_{1} \mathbf{S}_{1} \mathbf{B S}_{1} \mathbf{M}_{1} \mathbf{B}^{-1}-\lambda \mathbf{I}_{q}\right||\mathbf{B}|=0
$$

$\lambda_{i}$ is an eigenvalue of $\mathbf{M}_{1} \mathbf{S}_{1} \mathbf{B} \mathbf{S}_{1} \mathbf{M}_{1} \mathbf{B}^{-1} . \mathbf{M}_{1} \mathbf{S}_{1} \mathbf{B} \mathbf{S}_{1} \mathbf{M}_{1} \mathbf{B}^{-1}$ has the same eigenvalues with $\mathbf{B}^{-1} \mathbf{M}_{1} \mathbf{S}_{1} \mathbf{B S} \mathbf{S}_{1} \mathbf{M}_{1}$. If $\mathbf{B}-\mathbf{M}_{1} \mathbf{S}_{1} \mathbf{B} \mathbf{S}_{1} \mathbf{M}_{1}$ is an nnd matrix, $\mathbf{Q}^{\top} \mathbf{B Q}-\mathbf{Q}^{\top} \mathbf{M}_{1} \mathbf{S}_{1} \mathbf{B} \mathbf{S}_{1} \mathbf{M}_{1} \mathbf{Q}=$ $\mathbf{I}_{q}-\boldsymbol{\Lambda}$ is an nnd matrix. Then $1-\lambda_{i} \geq 0$, so we get $\lambda_{\max }\left(\mathbf{B}^{-1} \mathbf{M}_{1} \mathbf{S}_{1} \mathbf{B} \mathbf{S}_{1} \mathbf{M}_{1}\right) \leq 1$.

Let $\lambda_{\max }\left(\mathbf{B}^{-1} \mathbf{M}_{1} \mathbf{S}_{1} \mathbf{B} \mathbf{S}_{1} \mathbf{M}_{1}\right) \leq 1$. Since $\mathbf{B}$ is pd and $\mathbf{M}_{1} \mathbf{S}_{1} \mathbf{B S}_{1} \mathbf{M}_{1}$ is a symmetric matrix, we have get

$$
\lambda_{q} \leq \frac{x^{\top} \mathbf{M}_{1} \mathbf{S}_{1} \mathbf{B} \mathbf{S}_{1} \mathbf{M}_{1} x}{x^{\top} \mathbf{B} x} \leq \lambda_{1},
$$

where $\lambda_{1}\left(\mathbf{B}^{-1} \mathbf{M}_{1} \mathbf{S}_{1} \mathbf{B} \mathbf{S}_{1} \mathbf{M}_{1}\right) \geq \ldots \geq \lambda_{q}\left(\mathbf{B}^{-1} \mathbf{M}_{1} \mathbf{S}_{1} \mathbf{B} \mathbf{S}_{1} \mathbf{M}_{1}\right)$ are the roots of $\left|\mathbf{M}_{1} \mathbf{S}_{1} \mathbf{B} \mathbf{S}_{1} \mathbf{M}_{1}-\lambda \mathbf{B}\right|=$ 0 . Then we get $x^{\top} \mathbf{M}_{1} \mathbf{S}_{1} \mathbf{B} \mathbf{S}_{1} \mathbf{M}_{1} x \leq x^{\top} \mathbf{B} x$, so $\mathbf{B}-\mathbf{M}_{1} \mathbf{S}_{1} \mathbf{B} \mathbf{S}_{1} \mathbf{M}_{1}$ is an nnd matrix. It is obvious that $\boldsymbol{\Delta}_{1}$ is nnd if and only if $\lambda_{\max }\left(\mathbf{B}^{-1} \mathbf{M}_{1} \mathbf{S}_{1} \mathbf{B} \mathbf{S}_{1} \mathbf{M}_{1}\right) \leq 1$. 
3.1.2. The comparison between the FOARL estimator and the FOARML estimator. For the superiority of $\hat{\boldsymbol{\beta}}_{r}(d)^{(1)}$ over $\hat{\boldsymbol{\beta}}_{r}^{(1)}$, we consider the matrix MSE difference $\boldsymbol{\Delta}_{2}=$ $\operatorname{MSE}\left[\hat{\boldsymbol{\beta}}_{r}^{(1)}\right]-\operatorname{MSE}\left[\hat{\boldsymbol{\beta}}_{r}(d)^{(1)}\right]$. In the case of $\mathbf{R} \boldsymbol{\beta}=\mathbf{r}$, the matrix MSE difference $\boldsymbol{\Delta}_{2}$ equals to

$$
\boldsymbol{\Delta}_{2}=a(\phi) \mathbf{M}_{0}-a(\phi) \mathbf{M}_{1} \mathbf{S}_{d} \mathbf{S}^{-1} \mathbf{S}_{d} \mathbf{M}_{1}-(d-1)^{2} \mathbf{M}_{1} \boldsymbol{\beta} \boldsymbol{\beta}^{\top} \mathbf{M}_{1} .
$$

The result can be presented by Theorem 3.2 .

3.2. Theorem. Under the conditions that $a(\phi)\left[\mathbf{M}_{0}-\mathbf{M}_{1} \mathbf{S}_{d} \mathbf{S}^{-1} \mathbf{S}_{d} \mathbf{M}_{1}\right]$ is nnd, the estimator $\hat{\boldsymbol{\beta}}_{r}(d)^{(1)}$ is superior to the estimator $\hat{\boldsymbol{\beta}}_{r}^{(1)}$ by the matrix MSE criterion if and only if

$$
(d-1)^{2} \boldsymbol{\beta}^{\top} \mathbf{M}_{1}\left(\mathbf{M}_{0}-\mathbf{M}_{1} \mathbf{S}_{d} \mathbf{S}^{-1} \mathbf{S}_{d} \mathbf{M}_{1}\right)^{-} \mathbf{M}_{1} \boldsymbol{\beta} \leq 1,
$$

when the restrictions are true.

Proof. Let $\mathbb{C}(\mathbf{D})$ denotes the column space of $\mathbf{D}$ where $\mathbf{D}=a(\phi)\left[\mathbf{M}_{0}-\mathbf{M}_{1} \mathbf{S}_{d} \mathbf{S}^{-1} \mathbf{S}_{d}\right.$ $\left.\mathbf{M}_{1}\right]$. Then if $(1-d) \mathbf{M}_{1} \boldsymbol{\beta} \in \mathbb{C}(\mathbf{D}), \boldsymbol{\Delta}_{2}$ is nnd if and only if $(d-1)^{2} \boldsymbol{\beta}^{\top} \mathbf{M}_{1} \mathbf{D}^{-} \mathbf{M}_{1} \boldsymbol{\beta} \leq 1$ where $\mathbf{D}^{-}$is a generalized inverse of $\mathbf{D}$ under the condition that $\mathbf{D}$ is nnd, see [18].

Since $\mathbf{C}\left(\mathbf{M}_{1} \mathbf{S}_{d} \mathbf{S}^{-1} \mathbf{S}_{d} \mathbf{M}_{1}\right) \subset \mathbb{C}\left(\mathbf{M}_{0}\right)$ and $\Re\left(\mathbf{M}_{1} \mathbf{S}_{d} \mathbf{S}^{-1} \mathbf{S}_{d} \mathbf{M}_{1}\right) \subset \Re\left(\mathbf{M}_{0}\right)$ where $\Re(\ldots)$ denotes the row space of a matrix, applying Theorem B.1 in Appendix B from Harville [6], and by following Özkale [16], the g-inverse of $\mathbf{D}$ can be written as

$$
\mathbf{D}^{-}=\frac{1}{a(\phi)}\left[\mathbf{M}_{0}^{-}+\mathbf{M}_{0}^{-} \mathbf{M}_{1}\left(\mathbf{S}_{d}^{-1} \mathbf{S S}_{d}^{-1}-\mathbf{M}_{1} \mathbf{M}_{0}^{-} \mathbf{M}_{1}\right)^{-} \mathbf{M}_{1} \mathbf{M}_{0}^{-}\right] .
$$

Furthermore, $-(d-1) \mathbf{M}_{1} \boldsymbol{\beta} \in \mathbb{C}(\mathbf{D})$ if and only if $-(d-1) \mathbf{M}_{1} \boldsymbol{\beta}=-(d-1) \mathbf{D} \mathbf{D}^{-} \mathbf{M}_{1} \boldsymbol{\beta}$. The proof of the equality $-(d-1) \mathbf{M}_{1} \boldsymbol{\beta}=-(d-1) \mathbf{D} \mathbf{D}^{-} \mathbf{M}_{1} \boldsymbol{\beta}$ can be followed from Özkale [16].

3.1.3. The comparison between the FOARL estimator and the FOAML estimator. We consider the difference $\boldsymbol{\Delta}_{3}=\operatorname{MSE}\left[\hat{\boldsymbol{\beta}}^{(1)}\right]-\operatorname{MSE}\left[\hat{\boldsymbol{\beta}}_{r}(d)^{(1)}\right]=a(\phi) \mathbf{S}^{-1}-a(\phi) \mathbf{M}_{1} \mathbf{S}_{d} \mathbf{S}^{-1} \mathbf{S}_{d} \mathbf{M}_{1}-$ $(d-1)^{2} \mathbf{M}_{1} \boldsymbol{\beta} \boldsymbol{\beta}^{\top} \mathbf{M}_{1}$ to compare the FOAML estimator and the FOARL estimator. Then, we give Theorem 3.3 .

3.3. Theorem. Under the condition that $a(\phi)\left(\mathbf{S}^{-1}-\mathbf{M}_{1} \mathbf{S}_{d} \mathbf{S}^{-1} \mathbf{S}_{d} \mathbf{M}_{1}\right)$ is nnd, $\hat{\boldsymbol{\beta}}_{r}(d)^{(1)}$ is superior to $\hat{\boldsymbol{\beta}}^{(1)}$ in the sense of MSE matrix criterion if and only if

$$
(d-1)^{2} \boldsymbol{\beta}^{\top} \mathbf{M}_{1}\left(\mathbf{S}^{-1}-\mathbf{M}_{1} \mathbf{S}_{d} \mathbf{S}^{-1} \mathbf{S}_{d} \mathbf{M}_{1}\right)^{-} \mathbf{M}_{1} \boldsymbol{\beta} \leq a(\phi),
$$

when the restrictions are true.

Proof. Let $\mathbb{C}(\mathbf{A})$ denotes the column space of $\mathbf{A}$ where $\mathbf{A}=a(\phi)\left[\mathbf{S}^{-1}-\mathbf{M}_{1} \mathbf{S}_{d} \mathbf{S}^{-1} \mathbf{S}_{d}\right.$ $\left.\mathbf{M}_{1}\right]$. Then if $(1-d) \mathbf{M}_{1} \boldsymbol{\beta} \in \mathbb{C}(\mathbf{A}), \boldsymbol{\Delta}_{3}$ is nnd if and only if $(d-1)^{2} \boldsymbol{\beta}^{\top} \mathbf{M}_{1} \mathbf{A}^{-} \mathbf{M}_{1} \boldsymbol{\beta} \leq a(\phi)$ where $\mathbf{A}^{-}$is a g-inverse of $\mathbf{A}$ under the condition that $\mathbf{A}$ is nnd (see [18]).

Since $\mathbb{C}\left(\mathbf{M}_{1} \mathbf{S}_{d} \mathbf{S}^{-1} \mathbf{S}_{d} \mathbf{M}_{1}\right) \subset \mathbb{C}\left(\mathbf{S}^{-1}\right)$ and $\Re\left(\mathbf{M}_{1} \mathbf{S}_{d} \mathbf{S}^{-1} \mathbf{S}_{d} \mathbf{M}_{1}\right) \subset \Re\left(\mathbf{S}^{-1}\right)$ where $\Re(\cdot)$ denotes the row space of a matrix, applying Theorem B.1 in Appendix B from Harville [6], and by following Özkale [16], the g-inverse of A can be written as

$$
\mathbf{A}^{-}=\frac{1}{a(\phi)}\left[\mathbf{S}+\mathbf{S M}_{1}\left(\mathbf{S}_{d}^{-1} \mathbf{S S}_{d}^{-1}-\mathbf{M}_{1} \mathbf{S} \mathbf{M}_{1}\right)^{-} \mathbf{M}_{1} \mathbf{S}\right]
$$

Furthermore, $-(d-1) \mathbf{M}_{1} \boldsymbol{\beta} \in \mathbb{C}(\mathbf{A})$ if and only if $-(d-1) \mathbf{M}_{1} \boldsymbol{\beta}=-(d-1) \mathbf{A} \mathbf{A}^{-} \mathbf{M}_{1} \boldsymbol{\beta}$. The proof of the equality $-(d-1) \mathbf{M}_{1} \boldsymbol{\beta}=-(d-1) \mathbf{A} \mathbf{A}^{-} \mathbf{M}_{1} \boldsymbol{\beta}$ can be followed from Özkale [16].

\subsection{Comparisons when the restrictions are not true, i.e., $\mathbf{R} \beta \neq \mathbf{r}$.}


3.2.1. The comparison between the FOARL estimator and the FOARML estimator. In this case, the performances of the FOARL and FOARML estimators depend upon $\mathbf{R} \boldsymbol{\beta} \neq \mathbf{r}$ and both of them ore biased estimators of $\boldsymbol{\beta}$. We consider the difference

$$
\begin{aligned}
\boldsymbol{\Delta}_{4}= & \operatorname{MSE}\left[\hat{\boldsymbol{\beta}}_{r}^{(1)}\right]-\operatorname{MSE}\left[\hat{\boldsymbol{\beta}}_{r}(d)^{(1)}\right] \\
= & a(\phi)\left(\mathbf{M}_{0}-\mathbf{M}_{1} \mathbf{S}_{d} \mathbf{S}^{-1} \mathbf{S}_{d} \mathbf{M}_{1}\right)+\mathbf{S}^{-1} \mathbf{R}^{\top}\left(\mathbf{R} \mathbf{S}^{-1} \mathbf{R}^{\top}\right)^{-1}(\mathbf{R} \boldsymbol{\beta}-\mathbf{r})(\mathbf{R} \boldsymbol{\beta}-\mathbf{r})^{\top} \\
& \times\left(\mathbf{R} \mathbf{S}^{-1} \mathbf{R}^{\top}\right)^{-1} \mathbf{R}^{\top} \mathbf{S}^{-1}-(d-1)^{2}\left[\mathbf{M}_{1} \boldsymbol{\beta}-\mathbf{S}_{1}^{-1} \mathbf{R}^{\top}\left(\mathbf{R S}_{1}^{-1} \mathbf{R}^{\top}\right)^{-1}\right] \\
& \times(\mathbf{R} \boldsymbol{\beta}-\mathbf{r})(\mathbf{R} \boldsymbol{\beta}-\mathbf{r})^{\top}\left[\mathbf{M}_{1} \boldsymbol{\beta}-\mathbf{S}_{1}^{-1} \mathbf{R}^{\top}\left(\mathbf{R} \mathbf{S}_{1}^{-1} \mathbf{R}^{\top}\right)^{-1}\right]^{\top}
\end{aligned}
$$

to compare the FOARML estimator and the FOARL estimator. Then, we give Theorem 3.4 .

3.4. Theorem. Under the condition that $a(\phi)\left(\mathbf{M}_{0}-\mathbf{M}_{1} \mathbf{S}_{d} \mathbf{S}^{-1} \mathbf{S}_{d} \mathbf{M}_{1}\right)$ is an nnd matrix, the FOARL estimator dominates the FOARML estimator in terms of the matrix MSE criterion if and only if

$$
\begin{aligned}
& {\left[\boldsymbol{\beta}-\left(\mathbf{R} \mathbf{R}^{\top}\right)^{-1} \mathbf{r}\right]^{\top}\left(\mathbf{M}_{1} \mathbf{S}_{d}-\mathbf{M}_{0} \mathbf{S}\right)^{\top}\left(\mathbf{M}_{0}-\mathbf{M}_{1} \mathbf{S}_{d} \mathbf{S}^{-1} \mathbf{S}_{d} \mathbf{M}_{1}\right)^{-}} \\
& \times\left(\mathbf{M}_{1} \mathbf{S}_{d}-\mathbf{M}_{0} \mathbf{S}\right)\left[\boldsymbol{\beta}-\left(\mathbf{R} \mathbf{R}^{\top}\right)^{-1} \mathbf{r}\right]=0
\end{aligned}
$$

when the restrictions are incorrect.

Proof. $\boldsymbol{\Delta}_{4}$ can be written as $\boldsymbol{\Delta}_{4}=\mathbf{D}+\mathbf{d}_{1} \mathbf{d}_{1}^{\top}-\mathbf{d}_{2} \mathbf{d}_{2}^{\top}$ where $\mathbf{d}_{1}$ and $\mathbf{d}_{2}$ are the biases of the FOARML and FOARL estimators. To the end of the comparison, it is important whether $\mathbf{d}_{i} \in \mathbb{C}(\mathbf{D}), i=1,2$ or not. From Theorem B.3 in Appendix B $\mathbf{d}_{1} \notin \mathbb{C}(\mathbf{D})$, $\mathbf{d}_{2} \notin \mathbb{C}(\mathbf{D})$ and $\mathbf{d}_{2} \in \mathbb{C}\left(\mathbf{D}: \mathbf{d}_{1}\right)$. $\mathbf{d}_{1}$ and $\mathbf{d}_{2}$ are linearly independent. Thus when $\mathbf{D}$ is nnd, $\boldsymbol{\Delta}_{4}$ is nnd. Because of similarity between the FOARL and FOARML estimators in GLMs and restricted Liu and restricted ML estimators in linear model, the proof can be followed from Özkale [16].

3.2.2. The comparison between the FOARL estimator and the FOAML estimator. In this case, $\boldsymbol{\Delta}_{5}$ equals to

$$
\begin{aligned}
\boldsymbol{\Delta}_{5}= & \operatorname{MSE}\left[\hat{\boldsymbol{\beta}}^{(1)}\right]-\operatorname{MSE}\left[\hat{\boldsymbol{\beta}}_{r}(d)^{(1)}\right] \\
= & a(\phi) \mathbf{S}^{-1}-a(\phi) \mathbf{M}_{1} \mathbf{S}_{d} \mathbf{S}^{-1} \mathbf{S}_{d} \mathbf{M}_{1}-\left(\mathbf{M}_{1} \mathbf{S}_{d}-\mathbf{I}\right) \\
& \times\left[\boldsymbol{\beta}-\mathbf{R}^{\top}\left(\mathbf{R} \mathbf{R}^{\top}\right)^{-1} \mathbf{r}\right]\left[\boldsymbol{\beta}-\mathbf{R}^{\top}\left(\mathbf{R} \mathbf{R}^{\top}\right)^{-1} \mathbf{r}\right]^{\top}\left(\mathbf{M}_{1} \mathbf{S}_{d}-\mathbf{I}\right)^{\top} .
\end{aligned}
$$

to compare the FOARL estimator and the FOAML estimator. Then, we give Theorem 3.5 .

3.5. Theorem. Under the condition that $a(\phi)\left(\mathbf{S}^{-1}-\mathbf{M}_{1} \mathbf{S}_{d} \mathbf{S}^{-1} \mathbf{S}_{d} \mathbf{M}_{1}\right)$ is nnd matrix, the FOARL estimator is better than the FOAML estimator in the sense of the matrix $M S E$ criterion if and only if

$$
\begin{aligned}
& {\left[\boldsymbol{\beta}-\mathbf{R}^{\top}\left(\mathbf{R} \mathbf{R}^{\top}\right)^{-1} \mathbf{r}\right]^{\top}\left(\mathbf{M}_{1} \mathbf{S}_{d}-\mathbf{I}\right)^{\top}\left(\mathbf{S}^{-1}-\mathbf{M}_{1} \mathbf{S}_{d} \mathbf{S}^{-1} \mathbf{S}_{d} \mathbf{M}_{1}\right)^{-}} \\
& \times\left(\mathbf{M}_{1} \mathbf{S}_{d}-\mathbf{I}\right)\left[\boldsymbol{\beta}-\mathbf{R}^{\top}\left(\mathbf{R} \mathbf{R}^{\top}\right)^{-1} \mathbf{r}\right] \leq a(\phi)
\end{aligned}
$$

when the restrictions are not true.

Proof. If $\left(\mathbf{M}_{1} \mathbf{S}_{d}-\mathbf{I}\right)\left[\boldsymbol{\beta}-\mathbf{R}^{\top}\left(\mathbf{R R}^{\top}\right)^{-1} \mathbf{r}\right] \in \mathbb{C}(\mathbf{A})$ where $\mathbf{A}=a(\phi)\left[\mathbf{S}^{-1}-\mathbf{M}_{1} \mathbf{S}_{d} \mathbf{S}^{-1} \mathbf{S}_{d}\right.$ $\left.\mathbf{M}_{1}\right], \boldsymbol{\Delta}_{5}$ is nnd if and only if $\mathbf{A} \mathbf{A}^{-}\left(\mathbf{M}_{1} \mathbf{S}_{d}-\mathbf{I}\right)=\mathbf{M}_{1} \mathbf{S}_{d}-\mathbf{I}$. The proof of the equality $\mathbf{A A}^{-}\left(\mathbf{M}_{1} \mathbf{S}_{d}-\mathbf{I}\right)=\mathbf{M}_{1} \mathbf{S}_{d}-\mathbf{I}$ can be followed from Özkale [16].

\section{Numerical Examples}

We illustrate the theoretical results of the proposed estimator on two different real life data sets. 
4.1. Example 1: Mine Data Set. In this numerical example, the response has Poisson distribution with log link. The data set was originally given by Myers [14] and was also used by Marx [11]. Myers [14] presented 44 observations on mines in the coal fields of the Appalachian region of western Virginia. There are four continuous explanatory variables. These variables were analyzed for roles contributing to the number of injuries or fractures that occur in the upper seams of the mines.

In this study, as $[14,11]$ did, a generalized linear regression assuming the $y_{i}$ observations are from Poisson distribution and the log link function is going to be considered. The regression model is

$$
\log \hat{\mu}_{i}=\hat{\beta}_{0}+\hat{\beta}_{1} x_{1 i}+\hat{\beta}_{2} x_{2 i}+\hat{\beta}_{3} x_{3 i}+\hat{\beta}_{4} x_{4 i},
$$

where $\hat{\mu}_{i}$ is the expected number of upper seam injuries or fractures in the $i$ th coal mine area, $x_{1 i}$ is the corresponding inner burden thickness, which is the shortest distance between seam floor and lower seam, $x_{2 i}$ is the percent extraction of the lower previously mined seam, $x_{3 i}$ is the lower seam height and $x_{4 i}$ is the time that the mine has been opened.

Our computations here were performed by using $\mathrm{R}$. We used $1 \times 10^{-6}$ (sufficiently close to zero) as a convergence criterion. If the sum of absolute difference for the parameter estimates between the iterations is smaller than $1 \times 10^{-6}$, the iterations stop. The eigenvalues of $\mathbf{X}^{\top} \hat{\mathbf{W}} \mathbf{X}$ at final iteration of IRLS method are obtained as $\lambda_{1}=416468$, $\lambda_{2}=338966, \lambda_{3}=30607.8, \lambda_{4}=3824.86, \lambda_{5}=0.95040$. Thus, by following [11] the condition indices of the information matrix are 1.0000, 3.5052, 11.6647, 32.9976 and 2093.3225, indicating severe ill conditions and justifying an optional estimation technique.

To make comparisons, all the estimators are obtained by the first-order approximation. The ordinary least square (ls) estimator $\hat{\boldsymbol{\beta}}_{l s}=\left(\mathbf{X}^{\top} \mathbf{X}\right)^{-1} \mathbf{X}^{\top} \mathbf{y}$ is used as an initial value of $\boldsymbol{\beta}$ in computing the estimators and calculated as $\hat{\boldsymbol{\beta}}^{(0)}=(-4.579885,-0.001896,0.104574$, $-0.007993,-0.050250)^{\top}$. We calculate the weight matrix $\hat{\mathbf{W}}^{(0)}$ with the diagonal element $\hat{\mu}_{i}^{(0)}$, where $\hat{\mu}_{i}^{(0)}$ is $\exp \left(x \beta^{(0)}\right)$. The working response for Poisson distribution is defined as $\hat{z}_{i}^{(0)}=x_{i}^{\top} \beta^{(0)}+\left(y_{i}-\mu_{i}^{(0)}\right) / \mu_{i}^{(0)}, i=1, \ldots, n$.

The Liu-biasing parameter $d$ is computed as $\hat{d}_{h}$ which is proposed by Kurtoğlu and Özkale [8]. Therefore, according to the conditions given by Kurtoğlu and Özkale [8], $\hat{d}_{h}$ value is arbitrarily chosen as 0.95 .

The values of $\boldsymbol{\beta}$ affect the value of $\mathbf{R} \boldsymbol{\beta}-\mathbf{r}$ which measures the relative performances of the estimators. Therefore, to see how the restrictions affect the MSE values, four different types of restrictions are used corresponding to:

(i) $4 \beta_{1}-\beta_{3}=0$ where the effect of the third explanatory variable on the linear predictor is four times the first explanatory variable

$\left(\mathbf{R}^{\top}=\left[\begin{array}{lllll}0 & 4 & 0 & -1 & 0\end{array}\right], r=0\right)$,

(ii) $4 \beta_{1}-\beta_{3}=0.01$ where the effect of the difference between four times the first explanatory variable and the third explanatory variable on the linear predictor is 0.01 . Here, the number 0.01 is arbitrarily chosen to make difference from the restriction given by (i)

$\left(\mathbf{R}^{\top}=\left[\begin{array}{ccccc}0 & 4 & 0 & -1 & 0\end{array}\right], r=0.01\right)$,

(iii) $\beta_{0}+\beta_{1}+\beta_{4}=-5$ which means that sum of the effects of the constant, first and fourth explanatory variables on the linear predictor is -5

$\left(\mathbf{R}^{\top}=\left[\begin{array}{lllll}1 & 1 & 0 & 0 & 1\end{array}\right], r=-5\right)$,

(iv) $\beta_{0}+\beta_{1}+\beta_{4}=-5.5$ which means that sum of the effects of the constant, first and fourth explanatory variables on the linear predictor is -5.5 where the number -5.5 is arbitrarily chosen to make difference from the restriction given by (iii) $\left(\mathbf{R}^{\top}=\left[\begin{array}{lllll}1 & 1 & 0 & 0 & 1\end{array}\right], r=-5.5\right)$. 
Table 1. The parameter estimates and scalar MSE values of the estimators when $d=0.95$.

\begin{tabular}{cccccccr}
\hline Estimator & Rest. & \multicolumn{1}{c}{$\beta_{0}$} & \multicolumn{1}{c}{$\beta_{1}$} & \multicolumn{1}{c}{$\beta_{2}$} & \multicolumn{1}{c}{$\beta_{3}$} & $\beta_{4}$ & Scalar MSE \\
\hline$\hat{\boldsymbol{\beta}}^{(1)}$ & - & -4.909674 & -0.001789 & 0.097199 & -0.007045 & -0.047203 & 0.232122 \\
$\hat{\boldsymbol{\beta}}(d)^{(1)}$ & - & -4.863429 & -0.001782 & 0.096665 & -0.007101 & -0.047090 & 0.229910 \\
$\hat{\boldsymbol{\beta}}_{r}^{(1)}$ & (i) & -4.903588 & -0.001781 & 0.097159 & -0.007124 & -0.047166 & 0.209601 \\
& (ii) & -4.366989 & -0.001020 & 0.093567 & -0.014083 & -0.043899 & 0.504145 \\
& (iii) & -4.950893 & -0.001796 & 0.097676 & -0.006995 & -0.047310 & 0.001781 \\
& (iv) & -5.449518 & -0.001871 & 0.103437 & -0.006389 & -0.048609 & 0.291556 \\
$\hat{\boldsymbol{\beta}}_{r}(d)^{(1)}$ & (i) & -4.862113 & -0.001780 & 0.096658 & -0.007122 & -0.047080 & 0.172544 \\
& (ii) & -4.418463 & -0.001021 & 0.094188 & -0.014086 & -0.044005 & 0.411639 \\
& (iii) & -4.950882 & -0.001796 & 0.097676 & -0.006995 & -0.047321 & 0.001780 \\
& (iv) & -5.449489 & -0.001871 & 0.103438 & -0.006387 & -0.048639 & 0.291524 \\
Change $\%^{(1)}$ and $\left.\hat{\boldsymbol{\beta}}^{(d)}\right)^{(1)}$ & - & $\downarrow 0.94 \%$ & $\downarrow 0.38 \%$ & $\downarrow 0.54 \%$ & $\uparrow 0.79 \%$ & $\downarrow 0.23 \%$ & \\
$\hat{\boldsymbol{\beta}}_{r}^{(1)}$ & (i)-(ii) & $\downarrow 10.94 \%$ & $\downarrow 42.68 \%$ & $\downarrow 3.69 \%$ & $\uparrow 97.67 \%$ & $\downarrow 6.92 \%$ & \\
$\hat{\boldsymbol{\beta}}_{r}(d)^{(1)}$ & (iii)-(iv) & $\uparrow 10.07 \%$ & $\uparrow 4.19 \%$ & $\uparrow 5.89 \%$ & $\downarrow 8.66 \%$ & $\uparrow 2.74 \%$ & \\
& (i)-(ii) & $\downarrow 9.12 \%$ & $\downarrow 42.62 \%$ & $\downarrow 2.55 \%$ & $\uparrow 97.78 \%$ & $\downarrow 6.53 \%$ & \\
& (iii)-(iv) & $\uparrow 10.07 \%$ & $\uparrow 4.19 \%$ & $\uparrow 5.89 \%$ & $\downarrow 8.68 \%$ & $\uparrow 2.78 \%$ & \\
\hline
\end{tabular}
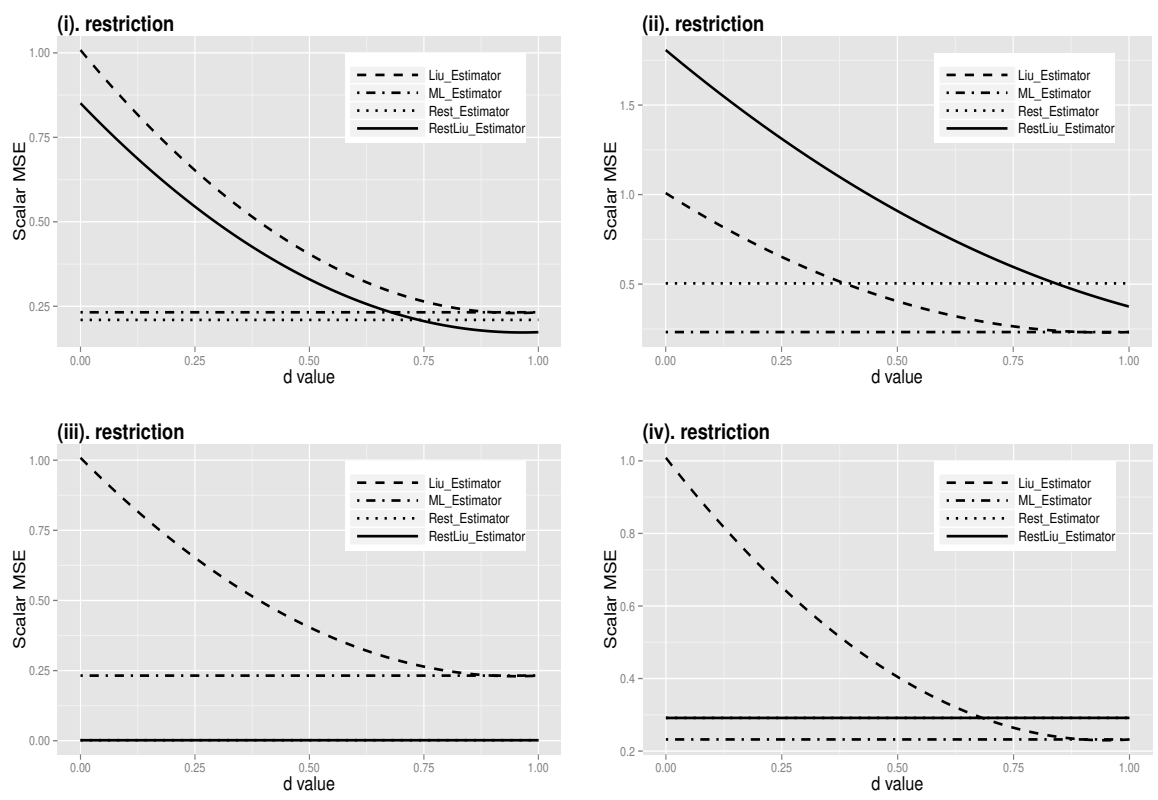

Figure 1. Scalar MSE values of the first-order approximated estimators versus $d$ under different restrictions (Mine Data Set).

The estimated parameter values, scalar MSE values and the change percentage in coefficients are given in Table 1 . The percentage change in $\boldsymbol{\beta}$ s are calculated as $\hat{\beta}_{i}=$ $\left[\left\{\hat{\beta}(\text { new })_{i}-\hat{\beta}(\text { old })_{i}\right\} / \hat{\beta}(\text { old })_{i}\right] \times 100 \%$ where $\hat{\boldsymbol{\beta}}$ shows any estimators. In computing the scalar MSE values, unknown $\boldsymbol{\beta}$ parameter vector is replaced by $\hat{\boldsymbol{\beta}}^{(1)}$ which is approximately unbiased (this idea is similar to those which are done the linear regression model). Table 1 shows that the estimators behave differently with respect to the model parameters and restrictions. For example, when the estimators are compared under restrictions (i)-(iii), it is observed that $\operatorname{sMSE}\left[\hat{\boldsymbol{\beta}}_{r}(d)^{(1)}\right]<\operatorname{sMSE}\left[\hat{\boldsymbol{\beta}}_{r}^{(1)}\right]<\operatorname{sMSE}\left[\hat{\boldsymbol{\beta}}(d)^{(1)}\right]<\operatorname{sMSE}\left[\hat{\boldsymbol{\beta}}^{(1)}\right]$ 


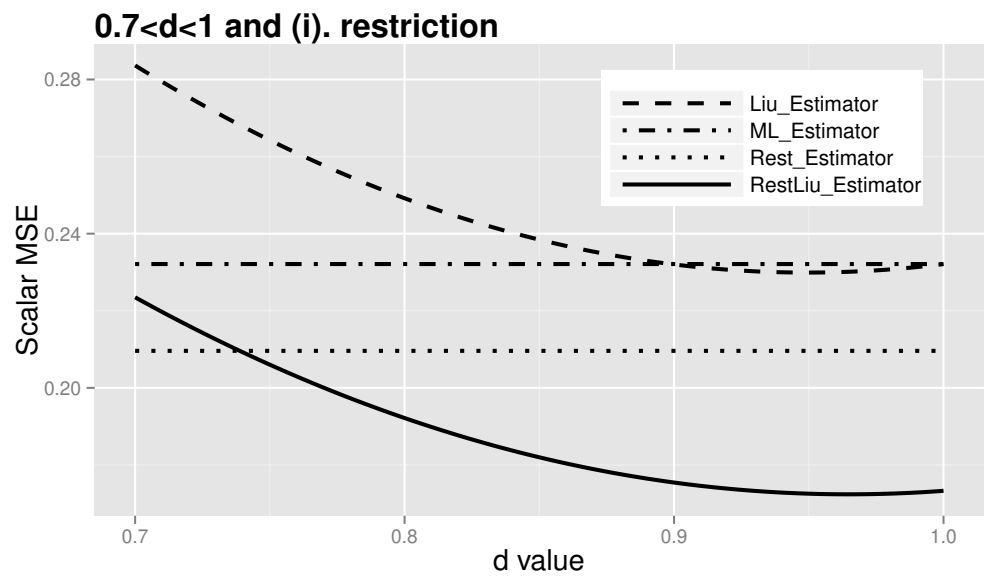

Figure 2. Scalar MSE values of the first-order approximated estimators versus $d$ in interval $(0.7,1)$ under restriction (i) (Mine Data Set).

under restriction (i), $\operatorname{sMSE}\left[\hat{\boldsymbol{\beta}}(d)^{(1)}\right]<\operatorname{sMSE}\left[\hat{\boldsymbol{\beta}}^{(1)}\right]<\operatorname{sMSE}\left[\hat{\boldsymbol{\beta}}_{r}(d)^{(1)}\right]<\operatorname{sMSE}\left[\hat{\boldsymbol{\beta}}_{r}^{(1)}\right]$ under restriction (ii), $\operatorname{sMSE}\left[\hat{\boldsymbol{\beta}}_{r}(d)^{(1)}\right]<\operatorname{sMSE}\left[\hat{\boldsymbol{\beta}}_{r}^{(1)}\right]<\operatorname{sMSE}\left[\hat{\boldsymbol{\beta}}(d)^{(1)}\right]<\operatorname{sMSE}\left[\hat{\boldsymbol{\beta}}^{(1)}\right]$ under restriction (iii) and $\operatorname{sMSE}\left[\hat{\boldsymbol{\beta}}(d)^{(1)}\right]<\operatorname{sMSE}\left[\hat{\boldsymbol{\beta}}^{(1)}\right]<\operatorname{sMSE}\left[\hat{\boldsymbol{\beta}}_{r}^{(1)}\right]<\operatorname{sMSE}\left[\hat{\boldsymbol{\beta}}_{r}(d)^{(1)}\right]$ under restriction (iv) where sMSE is the scalar MSE value.

There absolutely exists a biased estimator which outperforms $\hat{\boldsymbol{\beta}}^{(1)}$. It is observed that which estimator is superior than the other depends on the restriction and the Liubiasing parameter used. Under the (iii) and (iv) restrictions, the FOARML and FOARL estimators give almost the same scalar MSE values. As seen from Table 1, under the (i) and (iii) restrictions, the FOARML and FOARL estimators have smaller scalar MSE value than the FOAML and FOAL estimators.

The change in the percentage of coefficients between (i) and (ii) restrictions is maximum for the FOARML and FOARL estimators (approximately 97\%). The change in the percentage of coefficients between (i) and (ii) restrictions is minimum for the FOAML and FOAL estimators (approximately $0.2 \%$ ). In both the FOARML and FOARL estimators, the changes in percentage of the coefficients for (iii)-(iv) restrictions give the same amount of change in percentage.

To see the scalar MSE behavior of the estimators in GLMs for values of $d$ in the interval $(0,1)$, Figure 1 is given. To see the changes of the FOAL estimator more clearly in the scalar MSE values under (i) restriction, we have narrowed the interval of $d$ to $(0.7<d<1)$ in Figure 2. Figure 2 clearly indicates that as $d$ moves away from zero, scalar MSE of the FOAL is decreasing up to a $d$ value and then slightly increasing as $d$ approaches to one. Figure 2 suggests that the FOAL estimator has smaller scalar MSE value than the FOAML estimator when $d$ is larger than approximately 0.90. As $d$ moves away from 0.90 to 0 , the difference between the scalar MSE values of the FOAL and FOAML estimators increases where FOAML estimator gets better than FOAL estimator. Figure 1 also supports the results of Table 1 . In other words, since $d=0.95$ is larger than 0.90, the FOARL estimator has less scalar MSE value than the others as Figure 2 shows. As seen from Figure 1 that under restrictions (iii) and (iv), scalar MSE values of the FOARML and FOARL estimators are almost same (see Table 1 for $d=0.95$ ). As seen from Table 1 and Figure 1 that FOARML estimator performs better than FOAML 
estimator in the sense of scalar MSE criterion under the restrictions (i) and (iii). But it seen that FOARML estimator for the restrictions (ii) and (iv) is not superior over FOAML estimator in terms of scalar MSE. Figure 1 displays the behaviour of the scalar MSE value of FOAL estimator that $d$ gets larger (approaches 0.95), the scalar MSE value of FOAL estimator decreases more rapidly until $d=0.95$. After this value, the scalar MSE value of FOAL estimator increases slightly. As a consequence, we can say that the restrictions and the Liu-biasing parameter affect the performance of the estimators.

4.2. Example 2: Weather Data Set. In this numerical example, the response has gamma distribution with reciprocal link. The data set was first analyzed by Chatterjee and Hadi [3]. The data correspond to the weather factors and nitrogen dioxide concentrations $(y)$, in parts per hundred million (p.p.h.m.), for 26 days in September 1984 as measured at a monitoring station in the San Francisco Bay area. There are four explanatory variables. The variables considered in the study are: mean wind speed in miles per hour $\left(x_{1}\right)$ in m.p.h., maximum temperature $\left(x_{2}\right)$ in ${ }^{\circ} F$, insolation $\left(x_{3}\right)$ in langleys per day and stability factor $\left(x_{4}\right)$ in ${ }^{\circ} F$. Our computations here were performed by using $\mathrm{R}$. Chatterjee and Hadi [3] have shown that this data set has a gamma distribution.

The gamma model with reciprocal link is $\hat{\mu}_{i}=\left(\sum_{j=1}^{q} \hat{\beta}_{j} x_{i j}\right)^{-1}$. The Pearson estimate of $a(\phi)$ is obtained as $\hat{\varphi}^{2}=0.07572852$.

The constant term is added to the $\mathbf{X}$ matrix. The eigenvalues of the $\mathbf{X}^{\top} \hat{\mathbf{W}} \mathbf{X}$ matrix where $\hat{\mathbf{W}}$ is obtained at the final iteration of the IRLS method are obtained as $\lambda_{1}=$ 1240.428467, $\lambda_{2}=72.912574, \lambda_{3}=45.938929, \lambda_{4}=19.375420$, and $\lambda_{5}=5.801554$. Thus, the condition number (see [10]) which is computed as $\lambda_{\max } / \lambda_{\min }=213.8097$ indicates that multicollinearity exists.

To compute the IRLS estimator, we assigned with Hardin and Hilbe's [5] initial fitted values $\hat{\mu}_{i}^{(0)}=(y+\bar{y}) / 2$. Then we define the weight matrix $\mathbf{W}$ from $\left(\hat{\mathbf{W}}^{(0)}\right)^{-1}=$ $(d \boldsymbol{\eta} / d \boldsymbol{\mu})^{2} V^{(0)}$ and the working response $z$ with the $i$ th observation $\hat{z}_{i}^{(0)}=1 / \hat{\mu}_{i}^{(0)}-\left(y_{i}-\right.$ $\left.\hat{\mu}_{i}^{(0)}\right) /\left(\hat{\mu}_{i}^{(0)}\right)^{2}$ for the gamma distribution with reciprocal link.

All the estimators are obtained by the first-order approximation. The ordinary ls estimator $\hat{\boldsymbol{\beta}}_{l s}=\left(\mathbf{X}^{\top} \mathbf{X}\right)^{-1} \mathbf{X}^{\top} \mathbf{y}$ is used as an initial value of $\boldsymbol{\beta}$ and calculated as $\hat{\boldsymbol{\beta}}^{(0)}=$ $(6.269231,-5.622560,7.502692,-2.000586,-0.356589)^{\top}$.

Liu-biasing parameter $d$ is computed as $\hat{d}_{h}$ which is proposed by [8]. Therefore, according to the conditions given by [8], two $d_{h}$ values are arbitrarily chosen as 0.25 and 0.90 .

The restrictions are chosen as follows:

(i) $\beta_{0}+\beta_{1}+\beta_{2}+\beta_{3}+\beta_{4}=0$ which means that the sum of the effects of explanatory variables and the constant on the linear predictor is zero $\left(\mathbf{R}^{\top}=\left[\begin{array}{lllll}1 & 1 & 1 & 1 & 1\end{array}\right]\right.$, $r=0)$,

(ii) $\beta_{1}+\beta_{2}+\beta_{3}=0$ which means that sum of the effects of the explanatory variables

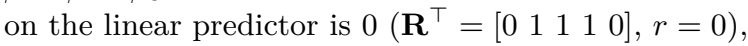

(iii) $\beta_{1}+\beta_{2}+\beta_{3}=0.01$ which means that sum of the effects of the explanatory variables on the linear predictor is 0.01 where the number $0.01^{\S}$ is arbitrarily chosen to make difference from the restriction given by (ii) $\left(\mathbf{R}^{\top}=\left[\begin{array}{lllll}0 & 1 & 1 & 1 & 0\end{array}\right]\right.$, $r=0.01)$.

Table 2 shows that the restrictions and the $\hat{d}_{h}$ values affect the MSE performance of the estimators. In computing the scalar MSE values, unknown $\boldsymbol{\beta}$ parameter vector is replaced by $\hat{\boldsymbol{\beta}}^{(1)}$ which is approximately unbiased. When the results in Table 2 are considered, it

§We recognized that as the deviation from 0 increases, scalar MSE values of the FOAL and FOARL estimators inflate. Therefore, 0.01 is arbitrarily choosen. 
Table 2. The parameter estimates and scalar MSE values of the estimators for different $d$ values and restrictions.

\begin{tabular}{cccrrrrrr}
\hline Estimator & $d$ & Rest. & \multicolumn{1}{c}{$\beta_{0}$} & \multicolumn{1}{c}{$\beta_{1}$} & \multicolumn{1}{c}{$\beta_{2}$} & \multicolumn{1}{c}{$\beta_{3}$} & \multicolumn{1}{c}{$\beta_{4}$} & Scalar MSE \\
\hline$\hat{\boldsymbol{\beta}}^{(1)}$ & - & - & 0.3089166 & 0.0132961 & -0.0037102 & 0.0000512 & 0.0000507 & 0.0417548 \\
$\hat{\boldsymbol{\beta}}(d)^{(1)}$ & 0.25 & - & 0.2267008 & 0.0150655 & -0.0029761 & 0.0000668 & -0.0003206 & 0.0292329 \\
& 0.90 & - & 0.2979545 & 0.0135320 & -0.0036123 & 0.0005332 & 0.00000012 & 0.0389611 \\
$\hat{\boldsymbol{\beta}}_{r}^{(1)}$ & - & (i) & -0.0181439 & 0.0202662 & -0.0007861 & 0.0001155 & -0.0014516 & 0.1070521 \\
& - & (ii) & 0.5720477 & 0.0059614 & -0.0060111 & 0.0000497 & 0.0010802 & 0.0761032 \\
$\hat{\boldsymbol{\beta}}_{r}(d)^{(1)}$ & - & (iii) & 0.2990107 & 0.0135723 & -0.0036235 & 0.0000513 & 0.0000120 & 0.0069032 \\
& 0.25 & (i) & -0.0181264 & 0.0202520 & -0.0007855 & 0.0001159 & -0.0014558 & 0.1070405 \\
& 0.25 & (ii) & 0.5312684 & 0.0055127 & -0.0056088 & 0.0000960 & 0.0007696 & 0.0555099 \\
& 0.25 & (iii) & 0.2807248 & 0.0133711 & -0.0034431 & 0.0000720 & -0.0001272 & 0.0067997 \\
& 0.90 & (i) & -0.0180952 & 0.0202273 & -0.0007844 & 0.0001166 & -0.0014642 & 0.1070197 \\
& 0.90 & (ii) & 0.5478234 & 0.0056949 & -0.0057722 & 0.0000772 & 0.0008957 & 0.0640246 \\
& 0.90 & (iii) & 0.2972799 & 0.0135532 & -0.0036065 & 0.0000532 & -0.0000011 & 0.0070209 \\
\hline
\end{tabular}
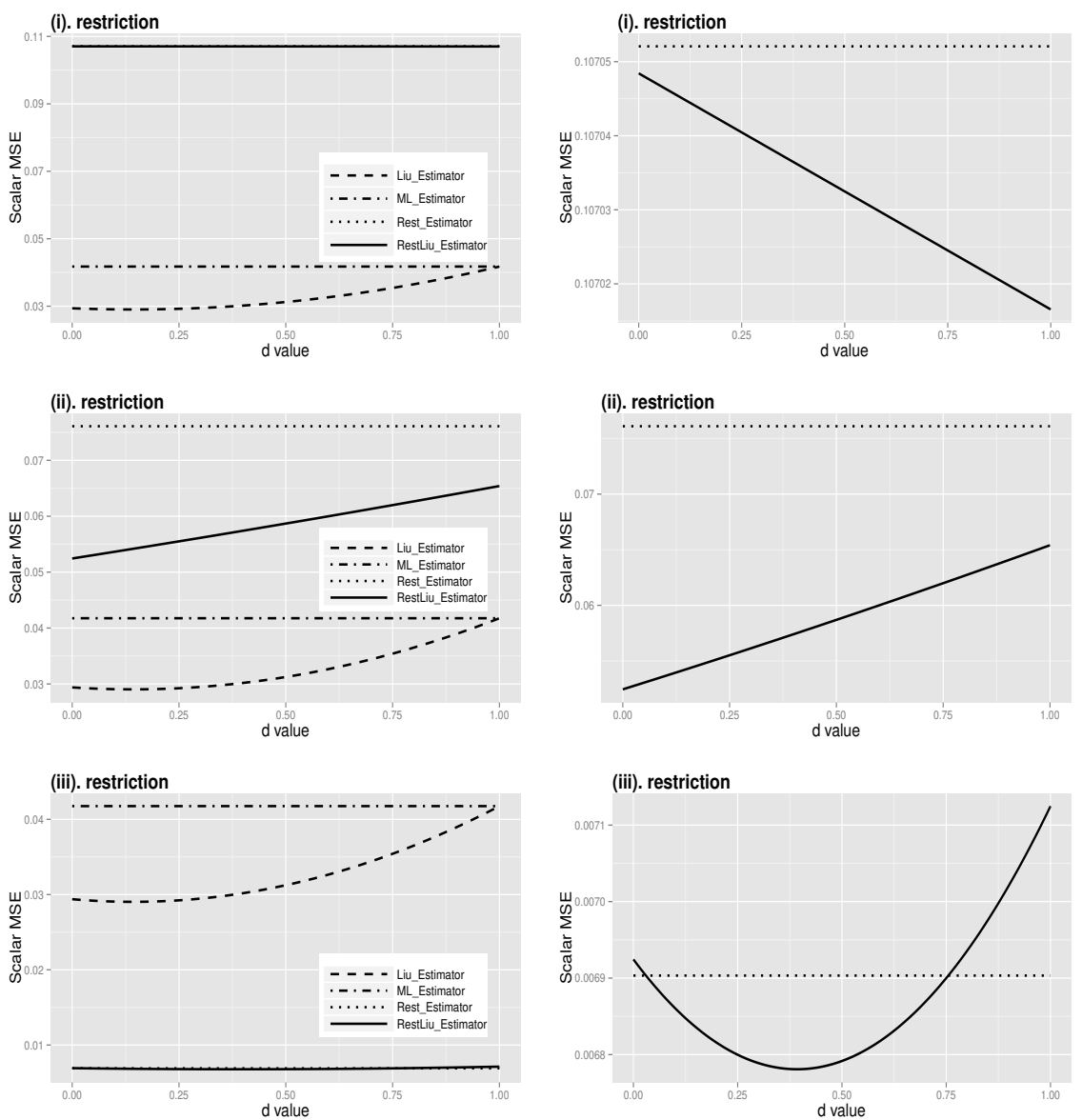

Figure 3. Scalar MSE values of the first-order approximated estimators versus $d$ under different restrictions (Weather Data Set). 
is observed that under restriction (i), the ordering $\operatorname{sMSE}\left[\hat{\boldsymbol{\beta}}(d=0.25)^{(1)}\right]<\operatorname{sMSE}[\hat{\boldsymbol{\beta}}(d=$ $\left.0.90)^{(1)}\right]<\operatorname{sMSE}\left[\hat{\boldsymbol{\beta}}^{(1)}\right]<\operatorname{sMSE}\left[\hat{\boldsymbol{\beta}}_{r}(d=0.90)^{(1)}\right]<\operatorname{sMSE}\left[\hat{\boldsymbol{\beta}}_{r}(d=0.25)^{(1)}\right]<\operatorname{sMSE}\left[\hat{\boldsymbol{\beta}}_{r}^{(1)}\right]$ exists. This ordering changes to $\operatorname{sMSE}\left[\hat{\boldsymbol{\beta}}(d=0.25)^{(1)}\right]<\operatorname{sMSE}\left[\hat{\boldsymbol{\beta}}(d=0.90)^{(1)}\right]<$ $\operatorname{sMSE}\left[\hat{\boldsymbol{\beta}}^{(1)}\right]<\operatorname{sMSE}\left[\hat{\boldsymbol{\beta}}_{r}(d=0.25)^{(1)}\right]<\operatorname{sMSE}\left[\hat{\boldsymbol{\beta}}_{r}(d=0.90)^{(1)}\right]<\operatorname{sMSE}\left[\hat{\boldsymbol{\beta}}_{r}^{(1)}\right]$ under restriction (ii), and $\operatorname{sMSE}\left[\hat{\boldsymbol{\beta}}_{r}(d=0.25)^{(1)}\right]<\operatorname{sMSE}\left[\hat{\boldsymbol{\beta}}_{r}^{(1)}\right]<\operatorname{sMSE}\left[\hat{\boldsymbol{\beta}}_{r}(d=0.90)^{(1)}\right]<$ $\operatorname{sMSE}\left[\hat{\boldsymbol{\beta}}(d=0.25)^{(1)}\right]<\operatorname{sMSE}\left[\hat{\boldsymbol{\beta}}(d=0.90)^{(1)}\right]<\operatorname{sMSE}\left[\hat{\boldsymbol{\beta}}^{(1)}\right]$ under restriction (iii).

In general, there absolutely exists a biased estimator which outperforms $\hat{\boldsymbol{\beta}}^{(1)}$. It is observed that which estimator is superior than the other depends on the restriction and the Liu-biasing parameter used.

To consider the effect of the restrictions and the Liu-biasing parameter on the estimators, the scalar MSE values of the estimators corresponding to $d$ values in the interval $(0,1)$ are given in Figure 3. Because of scaling, the performance of the FOARML and FOARL estimators are not obviously seen from the left side of Figure 3. Therefore, the scalar MSE values of the FOARML and FOARL estimators versus $d$ under all restrictions are plotted separately in the right side of Figure 3. As seen from Figure 3, as $d$ goes from 0 to 1 , the scalar MSE value of the FOAL estimator increases where as the scalar MSE value of the FOARL estimator decreases in linear manner under restriction (i), increase in linear manner under restriction (ii) and decrease and increase in a parabolic manner under restriction (iii). Figure 3 suggests that under restriction (iii), the FOARML and restricted Liu estimators perform better than the others. At restrictions (i) and (ii), the FOAL estimator shows better performance than the other estimators which is also supported by Table 2 .

\section{Monte Carlo simulation studies}

In this section, we examine the performance of the FOARL to the FOARML, FOAL and FOAML estimators in GLMs via simulation studies. Since the distribution of response, degree of multicollinearity, sample size, restriction types, and Liu-biasing parameter impact the performance of the estimators, simulation studies are conducted under various choices of these parameters. Our Monte Carlo simulation studies are performed by using $\mathrm{R}$ and the seed value of the generator of $\mathrm{R}$ is taken as 12345 . We design two simulation studies where one considers gamma response and the other Poisson response.

5.1. Experiment 1: Simulation study with gamma response. In this experiment, the response has gamma distribution with log link. The setting is as follows.

(1) To see the effect of the number of observations, the sample sizes are chosen as $n=100,200,300,400,500,600,700,800,900,1000$ and the number of explanatory variables is chosen as $q=4$.

(2) Following McDonald and Galarneau [13], the explanatory variables are generated by

$x_{i j}=\left(1-\gamma^{2}\right)^{1 / 2} w_{i j}+\gamma w_{i q+1}, i=1, \ldots, n, j=1, \ldots, q$,

where $w_{i j}$ are independent standard normal pseudo-random numbers and $\gamma$ is specified so that the correlation between any two explanatory variables is given by $\gamma^{2}$. The explanatory variables are then standardized by using unit length scaling so that $\mathbf{X}^{\top} \mathbf{X}$ is a matrix of correlations.

(3) To see the correlation effect in the simulation, we considered $\gamma^{2}=0.90,0.95$, 0.99 .

(4) For each set of explanatory variables, parameter vector $\boldsymbol{\beta}$ is chosen as $\boldsymbol{\beta}=$ $(1 / 2) \times j_{q \times 1}$ where $j_{q \times 1}$ is a $q \times 1$ vector of ones. 
(5) The values of $\boldsymbol{\beta}$ affect the value of $\mathbf{R} \boldsymbol{\beta}=\mathbf{r}$ which measures the correctness of the restrictions and the performances of the estimators depend on the magnitude of $\mathbf{R} \boldsymbol{\beta}=\mathbf{r}$. Therefore, following Özkale [16] four different scenarios are chosen:

(i) $\beta_{1}-2 \beta_{2}=0, \beta_{3}-2 \beta_{4}=0$ where the effect of the first explanatory variable on the linear predictor is twice the second explanatory and the effect of the third explanatory variable on the linear predictor is the fourth explanatory variable

$$
\left(\mathbf{R}^{\top}=\left[\begin{array}{cccc}
1 & -2 & 0 & 0 \\
0 & 0 & 1 & -2
\end{array}\right], \mathbf{r}=\left[\begin{array}{l}
0 \\
0
\end{array}\right]\right),
$$

(ii) $\beta_{1}+\beta_{2}+\beta_{3}+\beta_{4}=0$ where the sum of the effects of the explanatory variables on the linear predictor is $0\left(\mathbf{R}^{\top}=\left[\begin{array}{llll}1 & 1 & 1 & 1\end{array}\right], r=0\right)$,

(iii) $\beta_{1}-\beta_{2}=0, \beta_{3}-\beta_{4}=0$ where the first and second explanatory variables have same effect on the linear predictor, the third and fourth explanatory variables have the same effect on the linear predictor

$$
\left(\mathbf{R}^{\top}=\left[\begin{array}{cccc}
1 & -1 & 0 & 0 \\
0 & 0 & 1 & -1
\end{array}\right], \mathbf{r}=\left[\begin{array}{l}
0 \\
0
\end{array}\right]\right)
$$

(iv) $\beta_{1}-\beta_{2}=0, \beta_{3}-\beta_{4}=0, \beta_{1}-\beta_{3}=0$ where each explanatory variable affect the linear predictor in the same way

$$
\left(\mathbf{R}^{\top}=\left[\begin{array}{cccc}
1 & -1 & 0 & 0 \\
0 & 0 & 1 & -1 \\
1 & 0 & -1 & 0
\end{array}\right], \mathbf{r}=\left[\begin{array}{l}
0 \\
0 \\
0
\end{array}\right]\right)
$$

Note that in (i)-(ii), the restrictions are not true that is $\mathbf{R} \boldsymbol{\beta} \neq \mathbf{r}$ and in (iii)-(iv) the restrictions are indeed true that is $\mathbf{R} \boldsymbol{\beta}=\mathbf{r}$.

(6) The response of the gamma model is generated using pseudo-random numbers from $\operatorname{Gamma}\left(u=\hat{\mu}_{i}^{2} / \operatorname{var}_{i}, v=\operatorname{var}_{i} / \hat{\mu}_{i}\right)$ distribution with log link function where $\operatorname{var}_{i}=\hat{\mu}_{i}^{2}$ and

$$
\hat{\mu}_{i}=\exp \left(\hat{\beta}_{0}+\hat{\beta}_{1} x_{i 1}+\hat{\beta}_{2} x_{i 2}+\ldots+\hat{\beta}_{q} x_{i q}\right), \quad i=1, \ldots, n, j=1, \ldots, q .
$$

(7) Model parameters are estimated using the FOAML, FOARML and FOAL methods. We set the initial fitted values $\hat{\mu}_{i}^{(0)}=\exp \left(\mathbf{X} \hat{\boldsymbol{\beta}}^{(0)}\right)$ where $\hat{\boldsymbol{\beta}}^{(0)}$ is the ordinary ls estimator. We calculate the weight matrix $\hat{\mathbf{W}}^{(0)}$ with the diagonal element $\left(\hat{\mu}_{i}^{(0)}\right)^{2}$.

(8) We assign the working response $z$ with the $i$ th observation $\hat{z}_{i}^{(0)}=\log \left(\hat{\mu}_{i}^{(0)}\right)+$ $\left(y_{i}-\hat{\mu}_{i}^{(0)}\right) / \hat{\mu}_{i}^{(0)}$ for the gamma distribution.

(9) In the simulation procedure, the dispersion parameter is estimated to be the Pearson method $\hat{\varphi}_{p}^{2}=(n-q)^{-1} \sum_{i=1}^{n}\left(y_{i}-\hat{\mu}_{i}^{(0)}\right)^{2} /\left(\hat{\mu}_{i}^{(0)}\right)^{2}$ for each sample size and explanatory variables.

(10) The Liu-biasing parameters are chosen as $d=0.1,0.3,0.5,0.7$, and 0.9 .

(11) The number of replications in Monte Carlo simulation study is taken as 2000. The performance of the estimators is calculated in terms of the estimated MSE (EMSE):

$\operatorname{EMSE}(\tilde{\boldsymbol{\beta}})=\frac{1}{2000} \sum_{r=1}^{2000}\left(\tilde{\boldsymbol{\beta}}_{(r)}-\boldsymbol{\beta}\right)^{\top}\left(\tilde{\boldsymbol{\beta}}_{(r)}-\boldsymbol{\beta}\right)$,

where the subscript $(r)$ refers to the $r$ th replication and $\tilde{\boldsymbol{\beta}}_{(r)}$ is the estimate of $\boldsymbol{\beta}$ in the $r$ th replication of the experiment.

The simulation results are reported in Tables C1-C10. The main conclusions obtained from the simulation results are as follows:

(a) While the degree of collinearity becomes larger at fixed Liu-biasing parameter except for restriction (iv), the EMSE values of the estimators increase. 
(b) When the Liu-biasing parameter becomes larger at fixed degree of collinearity and sample size, the EMSE values of the FOAL and FOARL estimators increase.

(c) The sample sizes do not have an obvious effect on the EMSE values of the estimators at fixed degree of collinearity and Liu-biasing parameter.

(d) Taking account of restrictions, the difference between the EMSE values of the FOARL and FOARML estimators is least at the restrictions (iv). The largest difference of these estimators is at the restriction (ii).

(e) The Liu-biasing parameter, degree of collinearity and sample size do not have an obvious effect on these results.

5.2. Experiment 2: Simulation study with Poisson response. This experiment is designed mainly for Poisson distributed response. The explanatory variables are generated as in Experiment 1 and $n$ values, degree of correlation, Liu-biasing parameter $d$, parameter vector $\boldsymbol{\beta}$ and restrictions are chosen as in Experiment 1.

The $y_{i}$ observations are generated from a Poisson distribution $y_{i} \sim \mathcal{P}\left(\mu_{i}\right), i=1, \ldots, n$ where $\mu_{i}$ is determined by

$$
\hat{\mu}_{i}=\exp \left(\hat{\beta}_{1} x_{i 1}+\ldots+\hat{\beta}_{q} x_{i q}\right) .
$$

We calculate the weight matrix $\hat{\mathbf{W}}^{(0)}$ with the diagonal element $\hat{\mu}_{i}^{(0)}$ where $\hat{\mu}_{i}^{(0)}=$ $\exp \left(\mathbf{X} \hat{\boldsymbol{\beta}}^{(0)}\right)$. The working response for Poisson distribution is defined as $\hat{z}_{i}^{(0)}=x_{i}^{\top} \hat{\boldsymbol{\beta}}^{(0)}+$ $\left(y_{i}-\hat{\mu}_{i}^{(0)}\right) / \hat{\mu}_{i}^{(0)}, i=1, \ldots, n$.

The simulation results are reported in Tables C11-C20. The main conclusions obtained from the simulation results are as follows:

(a) The EMSE values of the FOAML and FOARML (except restriction (iv)) increase as the degree of correlation increases. This case is valid for the FOAL and FOARL estimators for fixed $d$ value; however, the increasement is not as severe as compared to the FOAL and FOARL estimators, respectively.

(b) As $d$ increases from 0.1 to 0.9 when correlation is fixed, the EMSE values of the FOAL and FOARL estimators increase.

(c) The sample sizes do not have an obvious effect on the EMSE values of all the estimators considered when correlation is fixed.

\section{Conclusions}

In this paper, to cope with the effects of multicollinearity in GLMs, a new estimator, called restricted Liu estimator in GLMs is introduced by unifying Liu and restricted ML estimators in GLMs. The superiority of the FOARL estimator over the FOAL, FOARML and FOAML estimators is examined with respect to the scalar MSE criterion. The numerical examples and simulation studies are conducted on the superiority of the FOARL estimator over the FOAML, FOARML, and FOAL estimators.

Based on the analyses, we conclude that the FOARL estimator outperforms the other estimators under certain restrictions. The Liu-biasing parameter affects the performance of the FOARL and FOAL estimators in GLMs.

\section{Appendix A. Derivation of the restricted Liu estimator}

Assuming that $\boldsymbol{\Lambda}^{*}$ and $\mathbf{X}^{\top} \mathbf{W X}+\mathbf{I}$ are invertible, application of inverse theorem (see p.428 [6]), we obtain

$$
\begin{aligned}
& \left\{\mathrm{E}\left[\mathrm{H}_{l}(\boldsymbol{\beta}, d, \boldsymbol{\lambda})\right]_{\beta=\hat{\beta}_{r}(d)}{ }^{(m)}\right\}^{-1}=a(\phi)\left\{\left(\mathbf{X}^{\top} \mathbf{W X}+\mathbf{I}\right)^{-1}-\left(\mathbf{X}^{\top} \mathbf{W X}+\mathbf{I}\right)^{-1}\right. \\
& \left.\times \mathbf{R}^{\top}\left[\left(\boldsymbol{\Lambda}^{*}\right)^{-1}+\mathbf{R}\left(\mathbf{X}^{\top} \mathbf{W X}+\mathbf{I}\right)^{-1} \mathbf{R}^{\top}\right]^{-1} \mathbf{R}\left(\mathbf{X}^{\top} \mathbf{W X}+\mathbf{I}\right)^{-1}\right\} .
\end{aligned}
$$


By using (2.2), (2.4) and (A.1), we get

$$
\begin{aligned}
& \mathrm{E}\left[\mathrm{H}_{l}(\boldsymbol{\beta}, d, \boldsymbol{\lambda})\right]_{\beta=\hat{\boldsymbol{\beta}}_{r}(d)^{(m)}}=\hat{\boldsymbol{\beta}}_{r}(d)^{(m)}+\left[\frac{\partial \psi(\boldsymbol{\beta}, d, \boldsymbol{\lambda})}{\partial \boldsymbol{\beta}}\right]_{\beta=\hat{\beta}_{r}(d)}(m) \\
& =\frac{1}{a(\phi)}\left[\left(\mathbf{X}^{\top} \mathbf{W} \mathbf{X}+\mathbf{I}\right) \hat{\boldsymbol{\beta}}_{r}(d)^{(m)}\right. \\
& \left.+\mathbf{X}^{\top} \mathbf{W} \mathbf{D}(\mathbf{y}-\boldsymbol{\mu})-\hat{\boldsymbol{\beta}}_{r}(d)^{(m)}+d \hat{\boldsymbol{\beta}}+\mathbf{R}^{\top} \boldsymbol{\Lambda}^{*} \mathbf{r}\right] .
\end{aligned}
$$

Following (A.1) and (A.2), $\hat{\boldsymbol{\beta}}_{r}(d)^{(m+1)}$ simplificies to

$$
\begin{aligned}
\hat{\boldsymbol{\beta}}_{r}(d)^{(m+1)=} & \left\{\left(\mathbf{X}^{\top} \mathbf{W} \mathbf{X}+\mathbf{I}\right)^{-1}-\left(\mathbf{X}^{\top} \mathbf{W} \mathbf{X}+\mathbf{I}\right)^{-1} \mathbf{R}^{\top}\right. \\
& \left.\times\left[\left(\boldsymbol{\Lambda}^{*}\right)^{-1}+\mathbf{R}\left(\mathbf{X}^{\top} \mathbf{W} \mathbf{X}+\mathbf{I}\right)^{-1} \mathbf{R}^{\top}\right]^{-1} \mathbf{R}\left(\mathbf{X}^{\top} \mathbf{W} \mathbf{X}+\mathbf{I}\right)^{-1}\right\} \\
& \times\left[\left(\mathbf{X}^{\top} \mathbf{W} \mathbf{X}+\mathbf{I}\right) \hat{\boldsymbol{\beta}}_{r}(d)^{(m)}+\mathbf{X}^{\top} \mathbf{W} \mathbf{D}(\mathbf{y}-\boldsymbol{\mu})-\hat{\boldsymbol{\beta}}_{r}(d)^{(m)}\right. \\
& \left.+d \hat{\boldsymbol{\beta}}+\mathbf{R}^{\top} \boldsymbol{\Lambda}^{*} \mathbf{r}\right]\left[\left(\mathbf{X}^{\top} \mathbf{W X}+\mathbf{I}\right)^{-1} \mathbf{R}^{\top} \boldsymbol{\Lambda}^{*} \mathbf{r}\right]
\end{aligned}
$$

After multiplying the terms and by using the following equation $\hat{\boldsymbol{\beta}}(d)^{(m+1)}=\left(\mathbf{X}^{\top} \mathbf{W} \mathbf{X}+\right.$ $\mathbf{I})^{-1}\left[\left(\mathbf{X}^{\top} \mathbf{W X}+\mathbf{I}\right) \hat{\boldsymbol{\beta}}_{r}(d)^{(m)}+\mathbf{X}^{\top} \mathbf{W D}(\mathbf{y}-\boldsymbol{\mu})-\hat{\boldsymbol{\beta}}_{r}(d)^{(m)}+d \hat{\boldsymbol{\beta}}\right]$, we get, after algebric simplifications,

$$
\begin{aligned}
\hat{\boldsymbol{\beta}}_{r}(d)^{(m+1)}= & \hat{\boldsymbol{\beta}}(d)^{(m+1)}-\left(\mathbf{X}^{\top} \mathbf{W} \mathbf{X}+\mathbf{I}\right)^{-1} \mathbf{R}^{\top}\left[\left(\boldsymbol{\Lambda}^{*}\right)^{-1}+\mathbf{R}\left(\mathbf{X}^{\top} \mathbf{W} \mathbf{X}+\mathbf{I}\right)^{-1}\right. \\
& \left.\times \mathbf{R}^{\top}\right]^{-1} \mathbf{R} \hat{\boldsymbol{\beta}}(d)^{(m+1)}+\left[\mathbf{I}-\left(\mathbf{X}^{\top} \mathbf{W} \mathbf{X}+\mathbf{I}\right)^{-1} \mathbf{R}\left[\left(\boldsymbol{\Lambda}^{*}\right)^{-1}\right.\right. \\
& \left.\left.+\mathbf{R}\left(\mathbf{X}^{\top} \mathbf{W} \mathbf{X}+\mathbf{I}\right)^{-1} \mathbf{R}^{\top}\right]^{-1} \mathbf{R}\right] \\
= & \hat{\boldsymbol{\beta}}(d)^{(m+1)}-\left(\mathbf{X}^{\top} \mathbf{W} \mathbf{X}+\mathbf{I}\right)^{-1} \mathbf{R}^{\top}\left[\left(\boldsymbol{\Lambda}^{*}\right)^{-1}+\mathbf{R}\left(\mathbf{X}^{\top} \mathbf{W X}+\mathbf{I}\right)^{-1}\right. \\
& \left.\times \mathbf{R}^{\top}\right]^{-1} \mathbf{R} \hat{\boldsymbol{\beta}}(d)^{(m+1)}+\left[\left(\mathbf{X}^{\top} \mathbf{W} \mathbf{X}+\mathbf{I}\right)+\mathbf{R}^{\top} \boldsymbol{\Lambda}^{*} \mathbf{r}\right]^{-1} \mathbf{R}^{\top} \boldsymbol{\Lambda}^{*} \mathbf{r} .
\end{aligned}
$$

For further simplifications and from (A.1), we obtain

$$
\begin{aligned}
& {\left[\left(\mathbf{X}^{\top} \mathbf{W X}+\mathbf{I}\right)+\mathbf{R}^{\top} \boldsymbol{\Lambda}^{*} \mathbf{r}\right]^{-1} \mathbf{R}^{\top} \boldsymbol{\Lambda}^{*} } \\
= & \left(\mathbf{X}^{\top} \mathbf{W} \mathbf{X}+\mathbf{I}\right)^{-1} \mathbf{R}^{\top} \boldsymbol{\Lambda}^{*}-\left(\mathbf{X}^{\top} \mathbf{W} \mathbf{X}+\mathbf{I}\right)^{-1} \mathbf{R}^{\top} \\
& \times\left[\left(\boldsymbol{\Lambda}^{*}\right)^{-1}+\mathbf{R}\left(\mathbf{X}^{\top} \mathbf{W} \mathbf{X}+\mathbf{I}\right)^{-1} \mathbf{R}^{\top}\right]^{-1} \mathbf{R}\left(\mathbf{X}^{\top} \mathbf{W} \mathbf{X}+\mathbf{I}\right)^{-1} \mathbf{R}^{\top} \boldsymbol{\Lambda}^{*} \\
= & \left(\mathbf{X}^{\top} \mathbf{W} \mathbf{X}+\mathbf{I}\right)^{-1} \mathbf{R}^{\top}\left[\left(\boldsymbol{\Lambda}^{*}\right)^{-1}+\mathbf{R}\left(\mathbf{X}^{\top} \mathbf{W} \mathbf{X}+\mathbf{I}\right)^{-1} \mathbf{R}^{\top}\right]^{-1} \\
& \times\left\{\left[\left(\boldsymbol{\Lambda}^{*}\right)^{-1}+\mathbf{R}\left(\mathbf{X}^{\top} \mathbf{W} \mathbf{X}+\mathbf{I}\right)^{-1} \mathbf{R}^{\top}\right]-\mathbf{R}\left(\mathbf{X}^{\top} \mathbf{W} \mathbf{X}+\mathbf{I}\right)^{-1} \mathbf{R}^{\top}\right\} \boldsymbol{\Lambda}^{*} \\
= & \left(\mathbf{X}^{\top} \mathbf{W} \mathbf{X}+\mathbf{I}\right)^{-1} \mathbf{R}^{\top}\left[\left(\boldsymbol{\Lambda}^{*}\right)^{-1}+\mathbf{R}\left(\mathbf{X}^{\top} \mathbf{W} \mathbf{X}+\mathbf{I}\right)^{-1} \mathbf{R}^{\top}\right]^{-1}\left(\boldsymbol{\Lambda}^{*}\right)^{-1} \boldsymbol{\Lambda}^{*} .
\end{aligned}
$$

Finally, $\hat{\boldsymbol{\beta}}_{r}(d)^{(m+1)}$ results in

$$
\begin{aligned}
\hat{\boldsymbol{\beta}}_{r}(d)^{(m+1)}= & \hat{\boldsymbol{\beta}}(d)^{(m+1)}-\left(\mathbf{X}^{\top} \mathbf{W} \mathbf{X}+\mathbf{I}\right)^{-1} \mathbf{R}^{\top}\left[\left(\boldsymbol{\Lambda}^{*}\right)^{-1}+\mathbf{R}\left(\mathbf{X}^{\top} \mathbf{W} \mathbf{X}+\mathbf{I}\right)^{-1}\right. \\
& \left.\times \mathbf{R}^{\top}\right]^{-1} \mathbf{R} \hat{\boldsymbol{\beta}}(d)^{(m+1)}+\left(\mathbf{X}^{\top} \mathbf{W} \mathbf{X}+\mathbf{I}\right)^{-1} \mathbf{R}^{\top}\left[\left(\boldsymbol{\Lambda}^{*}\right)^{-1}\right. \\
& \left.+\mathbf{R}\left(\mathbf{X}^{\top} \mathbf{W} \mathbf{X}+\mathbf{I}\right)^{-1} \mathbf{R}^{\top}\right]^{-1} \mathbf{r} \\
= & \hat{\boldsymbol{\beta}}(d)^{(m+1)}-\left(\mathbf{X}^{\top} \mathbf{W} \mathbf{X}+\mathbf{I}\right)^{-1} \\
& \times \mathbf{R}^{\top}\left[\left(\boldsymbol{\Lambda}^{*}\right)^{-1}+\mathbf{R}\left(\mathbf{X}^{\top} \mathbf{W} \mathbf{X}+\mathbf{I}\right)^{-1} \mathbf{R}^{\top}\right]^{-1}\left[\mathbf{R} \hat{\boldsymbol{\beta}}(d)^{(m+1)}-\mathbf{r}\right] .
\end{aligned}
$$

\section{Appendix B. Theorems}

B.1. Theorem. [6]] Let $\mathbf{R}$ represent an $n \times q$ matrix, $\mathbf{S}$ an $n \times m$ matrix, $\mathbf{T}$ an $m \times p$ matrix, and $\mathbf{U}$ a $p \times q$ matrix. If $\Re(\mathbf{S T U}) \subset \Re(\mathbf{R})$ and $\mathbb{C}(\mathbf{S T U}) \subset \mathbb{C}(\mathbf{R})$, then the matrix $\mathbf{R}^{-}-\mathbf{R}^{-} \mathbf{S} \mathbf{T} \mathbf{T}^{-}\left(\mathbf{T}^{-}+\mathbf{T}^{-} \mathbf{T} \mathbf{U} \mathbf{R}^{-} \mathbf{S} \mathbf{T} \mathbf{T}^{-}\right)^{-} \mathbf{T}^{-} \mathbf{T} \mathbf{U} \mathbf{R}^{-}$is a g-inverse of the matrix $\mathbf{R}+\mathbf{S T U}$

B.2. Theorem. ([4]) Let $\mathbf{A}$ and $\mathbf{B}$ be $n \times n$ symmetric matrices 
(1) If $\mathbf{A}$ is $p d$, there exists a nonsingular matrix $\mathbf{Q}$ such that $\mathbf{Q}^{\top} \mathbf{A Q}=\mathbf{I}$ and $\mathbf{Q}^{\top} \mathbf{B} \mathbf{Q}=\mathbf{D}$, where $\mathbf{D}$ is a diagonal matrix and the diagonal elements of $\mathbf{D}$ are roots $\boldsymbol{\lambda}$ of the polynomial equation $|\mathbf{B}-\boldsymbol{\lambda} \mathbf{A}|=0$.

(2) If $\mathbf{A}$ and $\mathbf{B}$ are both nonnegative (neither has to be positive definite), there exists a nonsingular matrix $\mathbf{Q}$ such that $\mathbf{Q}^{\top} \mathbf{A} \mathbf{Q}$ and $\mathbf{Q}^{\top} \mathbf{B} \mathbf{Q}$ are each diagonal.

B.3. Theorem. ([2]) Let $\boldsymbol{\varsigma}_{n \times p}$ be the set of $n \times p$ complex matrices and let $\mathbf{H}_{n}$ be the subset of $\boldsymbol{\varsigma}_{n \times n}$ consisting of Hermitian matrices. Further, given $\mathbf{L} \in \boldsymbol{\varsigma}_{n \times p}$, the symbols $\mathbf{L}^{*}, \mathbf{R}(\mathbf{L})$ and $\mathbf{\varsigma}(\mathbf{L})$ stand for the conjugate transpose, the range and set of all $g$-inverses, respectively of $\mathbf{L}$. Now, let $\mathbf{A} \in \mathbf{H}_{n}, \mathbf{a}_{1}$ and $\mathbf{a}_{2} \in \boldsymbol{\varsigma}_{n \times 1}$ be linearly independent, $\mathbf{f}_{i j}=$ $\mathbf{a}_{i}^{*} \mathbf{A}^{-} \mathbf{a}_{j}, i, j=1,2$ for $\mathbf{A}^{-} \in \boldsymbol{\varsigma}(\mathbf{A})$, and $\mathbf{s}=\left[\mathbf{a}_{1}^{*}\left(\mathbf{I}_{n}-\mathbf{A} \mathbf{A}^{-}\right)^{*}\left(\mathbf{I}_{n}-\mathbf{A} \mathbf{A}^{-}\right) \mathbf{a}_{2}\right] /\left[\mathbf{a}_{1}^{*}\left(\mathbf{I}_{n}-\right.\right.$ $\left.\left.\mathbf{A} \mathbf{A}^{-}\right)^{*}\left(\mathbf{I}_{n}-\mathbf{A A}^{-}\right) \mathbf{a}_{1}\right]$, provided that $\mathbf{a}_{1} \notin \mathbf{R}(\mathbf{A})$. Then $\mathbf{A}+\mathbf{a}_{1} \mathbf{a}_{1}^{*}-\mathbf{a}_{2} \mathbf{a}_{2}^{*}$ is nnd if and only if any one of the following sets of conditions hold.

(a) $\mathbf{A}$ is nnd, $\mathbf{a}_{1} \in \mathbf{R}(\mathbf{A}), \mathbf{a}_{2} \in \mathbf{R}(\mathbf{A})$ and $\left(\mathbf{f}_{11}+1\right)\left(\mathbf{f}_{22}-1\right) \leq\left|\mathbf{f}_{12}\right|^{2}$;

(b) $\mathbf{A}$ is $n n d, \mathbf{a}_{1} \notin \mathbf{R}(\mathbf{A}), \mathbf{a}_{2} \in \mathbf{R}\left(\mathbf{A}: \mathbf{a}_{1}\right)$ and $\left(\mathbf{a}_{2}-\mathbf{s} \mathbf{a}_{1}\right)^{*} \mathbf{A}^{-}\left(\mathbf{a}_{2}-\mathbf{s} \mathbf{a}_{1}\right) \leq 1-|\mathbf{s}|^{2}$;

(c) $\mathbf{A}=\mathbf{U} \Delta \mathbf{U}^{*}-\delta \mathbf{v} \mathbf{v}^{*}, \mathbf{a}_{1} \in \mathbf{R}(\mathbf{A}), \mathbf{a}_{2} \in \mathbf{R}(\mathbf{A}), \mathbf{v}^{*} a_{1} \neq 0$ and $\left(\mathbf{f}_{11}+1\right) \leq 0$, $\left(\mathbf{f}_{22}-1\right) \leq 0,\left(\mathbf{f}_{11}+1\right)\left(\mathbf{f}_{22}-1\right) \geq\left|\mathbf{f}_{12}\right|^{2}$ where $(\mathbf{U}: \mathbf{v})$ (with $\mathbf{U}$ possibly absent) is a subunitary matrix, $\boldsymbol{\Delta}$ is a pd diagonal matrix (occuring when $\mathbf{U}$ is present) and $\boldsymbol{\delta}$ is a positive scalar. Further, the conditions (a)-(c) are all independent of the choice of $\mathbf{A}^{-} \in \boldsymbol{\varsigma}(\mathbf{A})$.

\section{Appendix C. Tables of simulation studies}

Table C1: EMSEs of estimators for GLMs with gamma response when $\mathrm{n}=100$.

\begin{tabular}{|c|c|c|c|c|c|c|c|c|c|c|c|}
\hline \multirow[b]{3}{*}{$\mathrm{d}$} & \multirow[b]{3}{*}{ corr } & \multirow[t]{3}{*}{ FOAML } & \multicolumn{4}{|c|}{ FOARML } & \multirow{3}{*}{ FOAL } & \multicolumn{4}{|c|}{ FOARL } \\
\hline & & & \multicolumn{4}{|c|}{ Restrictions } & & \multicolumn{4}{|c|}{ Restrictions } \\
\hline & & & (i) & (ii) & (iii) & (iv) & & (i) & (ii) & (iii) & (iv) \\
\hline \multirow{3}{*}{0.1} & 0.90 & 29.5422 & 11.0529 & 30.3281 & 11.5347 & 0.2743 & 1.1915 & 0.6463 & 1.9655 & 0.5626 & 0.2267 \\
\hline & 0.95 & 59.4653 & 21.6902 & 60.2694 & 22.9911 & 0.2647 & 1.4184 & 0.7308 & 2.1978 & 0.6528 & 0.2208 \\
\hline & 0.99 & 301.0209 & 106.8740 & 301.8662 & 115.3358 & 0.2582 & 3.7647 & 1.5997 & 4.5479 & 1.5706 & 0.2169 \\
\hline \multirow{3}{*}{0.3} & 0.90 & 29.5422 & 11.0529 & 30.3281 & 11.5347 & 0.2743 & 4.0886 & 1.7324 & 4.8596 & 1.6718 & 0.2297 \\
\hline & 0.95 & 59.4653 & 21.6902 & 60.2694 & 22.9911 & 0.2647 & 6.7766 & 2.7276 & 7.5534 & 2.7145 & 0.2235 \\
\hline & 0.99 & 301.0209 & 106.8740 & 301.8662 & 115.3358 & 0.2582 & 28.5083 & 10.7601 & 29.2891 & 11.1787 & 0.2194 \\
\hline \multirow{3}{*}{0.5} & 0.90 & 29.5422 & 11.0529 & 30.3281 & 11.5347 & 0.2743 & 8.9303 & 3.5675 & 9.6937 & 3.5591 & 0.2370 \\
\hline & 0.95 & 59.4653 & 21.6902 & 60.2694 & 22.9911 & 0.2647 & 16.4440 & 6.3548 & 17.2141 & 6.4784 & 0.2302 \\
\hline & 0.99 & 301.0209 & 106.8740 & 301.8662 & 115.3358 & 0.2582 & 76.8595 & 28.6882 & 77.6340 & 30.0105 & 0.2257 \\
\hline \multirow{3}{*}{0.7} & 0.90 & 29.5422 & 11.0529 & 30.3281 & 11.5347 & 0.2743 & 15.7166 & 6.1515 & 16.4678 & 6.2244 & 0.2487 \\
\hline & 0.95 & 59.4653 & 21.6902 & 60.2694 & 22.9911 & 0.2647 & 30.4206 & 11.6123 & 31.1798 & 11.9444 & 0.2410 \\
\hline & 0.99 & 301.0209 & 106.8740 & 301.8662 & 115.3358 & 0.2582 & 148.8183 & 55.3839 & 149.5828 & 58.0658 & 0.2358 \\
\hline \multirow{3}{*}{0.9} & 0.90 & 29.5422 & 11.0529 & 30.3281 & 11.5347 & 0.2743 & 24.4475 & 9.4846 & 25.1821 & 9.6677 & 0.2648 \\
\hline & 0.95 & 59.4653 & 21.6902 & 60.2694 & 22.9911 & 0.2647 & 48.7065 & 18.5001 & 49.4506 & 19.1125 & 0.2558 \\
\hline & 0.99 & 301.0209 & 106.8740 & 301.8662 & 115.3358 & 0.2582 & 244,3848 & 90,8474 & 245.1353 & 95.3447 & 0.2498 \\
\hline
\end{tabular}


Table C2: EMSEs of estimators for GLMs with gamma response when $\mathrm{n}=200$.

\begin{tabular}{|c|c|c|c|c|c|c|c|c|c|c|c|}
\hline \multirow[b]{3}{*}{$\mathrm{d}$} & \multirow[b]{3}{*}{ corr } & \multirow{3}{*}{ FOAML } & \multicolumn{4}{|c|}{ FOARML } & \multirow{3}{*}{ FOAL } & \multicolumn{4}{|c|}{ FOARL } \\
\hline & & & \multicolumn{4}{|c|}{ Restrictions } & & \multicolumn{4}{|c|}{ Restrictions } \\
\hline & & & (i) & (ii) & (iii) & (iv) & & (i) & (ii) & (iii) & (iv) \\
\hline \multirow{3}{*}{0.1} & 0.90 & 30.1531 & 11.6998 & 30.8828 & 11.4509 & 0.2650 & 1.1771 & 0.6343 & 1.9630 & 0.5419 & 0.2140 \\
\hline & 0.95 & 59.7589 & 22.9170 & 60.4954 & 22.5290 & 0.2552 & 1.4040 & 0.7221 & 2.1958 & 0.6283 & 0.2082 \\
\hline & 0.99 & 295.7997 & 112.4884 & 296.5275 & 110.9072 & 0.2479 & 3.6976 & 1.5997 & 4.4937 & 1.4941 & 0.2039 \\
\hline \multirow{3}{*}{0.3} & 0.90 & 30.1531 & 11.6998 & 30.8828 & 11.4509 & 0.2650 & 4.1285 & 1.7485 & 4.9106 & 1.6345 & 0.2175 \\
\hline & 0.95 & 59.7589 & 22.9170 & 60.4954 & 22.5290 & 0.2552 & 6.7874 & 2.7575 & 7.5758 & 2.6311 & 0.2113 \\
\hline & 0.99 & 295.7997 & 112.4884 & 296.5275 & 110.9072 & 0.2479 & 28.0228 & 10.8483 & 28.8158 & 10.6216 & 0.2068 \\
\hline \multirow{3}{*}{0.5} & 0.90 & 30.1531 & 11.6998 & 30.8828 & 11.4509 & 0.2650 & 9.0729 & 3.6384 & 9.8467 & 3.4950 & 0.2255 \\
\hline & 0.95 & 59.7589 & 22.9170 & 60.4954 & 22.5290 & 0.2552 & 16.5047 & 6.4616 & 17.2856 & 6.2850 & 0.2187 \\
\hline & 0.99 & 295.7997 & 112.4884 & 296.5275 & 110.9072 & 0.2479 & 75.5401 & 28.9512 & 76.3263 & 28.5005 & 0.2136 \\
\hline \multirow{3}{*}{0.7} & 0.90 & 30.1531 & 11.6998 & 30.8828 & 11.4509 & 0.2650 & 16.0103 & 6.3038 & 16.7712 & 6.1235 & 0.2380 \\
\hline & 0.95 & 59.7589 & 22.9170 & 60.4954 & 22.5290 & 0.2552 & 30.5560 & 11.8342 & 31.3251 & 11.5899 & 0.2302 \\
\hline & 0.99 & 295.7997 & 112.4884 & 296.5275 & 110.9072 & 0.2479 & 146.2498 & 55.9084 & 147.0251 & 55.1307 & 0.2244 \\
\hline \multirow{3}{*}{0.9} & 0.90 & 30.1531 & 11.6998 & 30.8828 & 11.4509 & 0.2650 & 24.9406 & 9.7449 & 25.6842 & 9.5199 & 0.2549 \\
\hline & 0.95 & 59.7589 & 22.9170 & 60.4954 & 22.5290 & 0.2552 & 48.9411 & 18.8754 & 49.6943 & 18.5458 & 0.2458 \\
\hline & 0.99 & 295.7997 & 112.4884 & 296.5275 & 110.9072 & 0.2479 & 240.1517 & 91.7198 & 240.9122 & 90.5122 & 0.2391 \\
\hline
\end{tabular}

Table C3: EMSEs of estimators for GLMs with gamma response when $\mathrm{n}=300$.

\begin{tabular}{|c|c|c|c|c|c|c|c|c|c|c|c|}
\hline \multirow[b]{3}{*}{ d } & \multirow[b]{3}{*}{ corr } & \multirow[t]{3}{*}{ FOAML } & \multirow{2}{*}{\multicolumn{4}{|c|}{$\begin{array}{l}\text { FOARML } \\
\text { Restrictions }\end{array}$}} & \multirow[t]{3}{*}{ FOAL } & \multicolumn{4}{|c|}{ FOARL } \\
\hline & & & & & & & & & Restric & ions & \\
\hline & & & (i) & (ii) & (iii) & (iv) & & (i) & (ii) & (iii) & (iv) \\
\hline \multirow{3}{*}{0.1} & 0.90 & 29.2874 & 9.1568 & 30.0099 & 9.2613 & 0.2631 & 1.1714 & 0.6014 & 1.9570 & 0.5148 & 0.2142 \\
\hline & 0.95 & 58.7009 & 18.0131 & 59.4287 & 18.3725 & 0.2517 & 1.3928 & 0.6622 & 2.1859 & 0.5788 & 0.2067 \\
\hline & 0.99 & 295.3786 & 89.3077 & 296.0891 & 91.7421 & 0.2430 & 3.6899 & 1.3523 & 4.4890 & 1.2934 & 0.2009 \\
\hline \multirow{3}{*}{0.3} & 0.90 & 4 & 9.1568 & 30.0099 & 9.2613 & 0.2631 & 91 & 1.4915 & 4.8314 & 1.4170 & 0.2173 \\
\hline & 0.95 & 58.7009 & 18.0131 & 59.4287 & 18.3725 & 0.2517 & 6.6896 & 2.2856 & 7.4798 & 2.2377 & 0.2095 \\
\hline & 0.99 & 295.3786 & 89.3077 & 296.0891 & 91.7421 & 0.2430 & 27.9808 & 8.7243 & 28.7773 & 8.8954 & 0.2034 \\
\hline \multirow{3}{*}{0.5} & 0.90 & 29.28 & 9.1568 & 30.0099 & 9.2613 & 0.2631 & 8.8528 & 2.9642 & 9.6271 & 2.9228 & 0.2248 \\
\hline & 0.95 & 58.7009 & 18.0131 & 59.4287 & 18.3725 & 0.2517 & 16.2369 & 5.1944 & 17.0199 & 5.2271 & 0.2164 \\
\hline & 0.99 & 295.3786 & 89.3077 & 296.0891 & 91.7421 & 0.2430 & 75.4310 & 23.1019 & 76.2210 & 23.7442 & 0.2098 \\
\hline \multirow{3}{*}{0.7} & 0.90 & 29.2 & 9.1568 & 30.0099 & 9.2613 & 0.2631 & 15.5 & 5.0195 & 16.3441 & 5.0321 & 0.2368 \\
\hline & 0.95 & 58.7009 & 18.0131 & 59.4287 & 18.3725 & 0.2517 & 30.0347 & 9.3885 & 30.8063 & 9.5470 & 0.2274 \\
\hline & 0.99 & 295.3786 & 89.3077 & 296.0891 & 91.7421 & 0.2430 & 146.0406 & 44.4849 & 146.8201 & 45.8398 & 0.2201 \\
\hline \multirow{3}{*}{0.9} & 0.90 & 29.2 & 9.1568 & 30.0099 & 9.2613 & 0.2631 & 24.2 & 7.6575 & 24.9824 & 7.7450 & 0.2533 \\
\hline & 0.95 & 58.7009 & 18.0131 & 59.4287 & 18.3725 & 0.2517 & 48.0829 & 14.8680 & 48.8390 & 15.1974 & 0.2426 \\
\hline & 0.99 & 295.3786 & 89.3077 & 296.0891 & 91.7421 & 0.2430 & 239.8095 & 72.8734 & 240.5746 & 75.1823 & 0.2344 \\
\hline
\end{tabular}


Table C4: EMSEs of estimators for GLMs with gamma response when $\mathrm{n}=400$.

\begin{tabular}{|c|c|c|c|c|c|c|c|c|c|c|c|}
\hline \multirow[b]{3}{*}{ d } & \multirow[b]{3}{*}{ corr } & \multirow[t]{3}{*}{ FOAML } & \multirow{2}{*}{\multicolumn{4}{|c|}{$\begin{array}{c}\text { FOARML } \\
\text { Restrictions }\end{array}$}} & \multirow[t]{3}{*}{ FOAL } & \multicolumn{4}{|c|}{ FOARL } \\
\hline & & & & & & & & & Restric & ions & \\
\hline & & & (i) & (ii) & (iii) & (iv) & & (i) & (ii) & (iii) & (iv) \\
\hline \multirow{3}{*}{0.1} & 0.90 & 30.49 & 10.1679 & 31.2269 & 10.1423 & 0.2692 & 1.1982 & 0.6309 & 1.9880 & 0.5447 & 0.2102 \\
\hline & 0.95 & 60.7168 & 19.9452 & 61.4530 & 20.0150 & 0.2591 & 1.4280 & 0.7012 & 2.2239 & 0.6146 & 0.2040 \\
\hline & 0.99 & 302.5332 & 98.3480 & 303.2697 & 99.1015 & 0.2516 & 3.7765 & 1.4669 & 4.5772 & 1.3820 & 0.1993 \\
\hline \multirow{3}{*}{0.3} & 0.90 & 30.4 & 10.1679 & 31.2269 & 10.1423 & 0.2692 & 4.1 & 1.6256 & 4.9787 & 1.5367 & 0.2152 \\
\hline & 0.95 & 60.7168 & 19.9452 & 61.4530 & 20.0150 & 0.2591 & 6.9077 & 2.5100 & 7.6989 & 2.4243 & 0.2087 \\
\hline & 0.99 & 302.5332 & 98.3480 & 303.2697 & 99.1015 & 0.2516 & 28.6622 & 9.6375 & 29.4584 & 9.5716 & 0.2038 \\
\hline \multirow{3}{*}{0.5} & 0.90 & 30.49 & 10.1679 & 31.2269 & 10.1423 & 0.2692 & 9.1987 & 3.2757 & 9.9735 & 3.1866 & 0.2248 \\
\hline & 0.95 & 60.7168 & 19.9452 & 61.4530 & 20.0150 & 0.2591 & 16.7849 & 5.7552 & 17.5669 & 5.6759 & 0.2177 \\
\hline & 0.99 & 302.5332 & 98.3480 & 303.2697 & 99.1015 & 0.2516 & 77.2648 & 25.5768 & 78.0524 & 25.5526 & 0.2123 \\
\hline \multirow{3}{*}{0.7} & 0.90 & 30.49 & 10.1679 & 31.2269 & 10.1423 & 0.2692 & 16.2 & 5.5810 & 16.9725 & 5.4944 & 0.2391 \\
\hline & 0.95 & 60.7168 & 19.9452 & 61.4530 & 20.0150 & 0.2591 & 31.0595 & 10.4370 & 31.8281 & 10.3695 & 0.2310 \\
\hline & 0.99 & 302.5332 & 98.3480 & 303.2697 & 99.1015 & 0.2516 & 149.5844 & 49.2849 & 150.3594 & 49.3249 & 0.2249 \\
\hline \multirow{3}{*}{0.9} & 0.90 & 30.4989 & 10.1679 & 31.2269 & 10.1423 & 0.2692 & 25.2345 & 8.5417 & 25.9756 & 8.4600 & 0.2580 \\
\hline & 0.95 & 60.7168 & 19.9452 & 61.4530 & 20.0150 & 0.2591 & 49.7317 & 16.5552 & 50.4824 & 16.5050 & 0.2487 \\
\hline & 0.99 & 302.5332 & 98.3480 & 303.2697 & 99.1015 & 0.2516 & 245.6210 & 80.7618 & 246.3792 & 80.8885 & 0.2417 \\
\hline
\end{tabular}

Table C5: EMSEs of estimators for GLMs with gamma response when $\mathrm{n}=500$.

\begin{tabular}{|c|c|c|c|c|c|c|c|c|c|c|c|}
\hline \multirow[b]{3}{*}{$\mathrm{d}$} & \multirow[b]{3}{*}{ corr } & \multirow[t]{3}{*}{ FOAML } & \multirow{2}{*}{\multicolumn{4}{|c|}{$\begin{array}{l}\text { FOARML } \\
\text { Restrictions }\end{array}$}} & \multirow[t]{3}{*}{ FOAL } & \multicolumn{4}{|c|}{ FOARL } \\
\hline & & & & & & & & & Restric & tions & \\
\hline & & & (i) & (ii) & (iii) & (iv) & & (i) & (ii) & (iii) & (iv) \\
\hline \multirow{3}{*}{0.1} & 0.90 & 29.6030 & 10.4164 & 30.3499 & 10.0334 & 0.2697 & 1.1968 & 0.6330 & 1.9752 & 0.5455 & 0.2218 \\
\hline & 0.95 & 59.1960 & 20.5059 & 59.9570 & 19.8935 & 0.2588 & 1.4185 & 0.7065 & 2.2041 & 0.6151 & 0.2145 \\
\hline & 0.99 & 296.8833 & 101.5791 & 297.6659 & 99.1856 & 0.2506 & 3.7243 & 1.4973 & 4.5155 & 1.3830 & 0.2088 \\
\hline \multirow{3}{*}{0.3} & 0.90 & 29.6030 & 10.4164 & 30.3499 & 10.0334 & 0.2697 & 4.1111 & 1.6422 & 4.8867 & 1.5207 & 0.2248 \\
\hline & 0.95 & 59.1960 & 20.5059 & 59.9570 & 19.8935 & 0.2588 & 6.7674 & 2.5513 & 7.5505 & 2.4043 & 0.2172 \\
\hline & 0.99 & 296.8833 & 101.5791 & 297.6659 & 99.1856 & 0.2506 & 28.1492 & 9.8955 & 28.9380 & 9.5523 & 0.2113 \\
\hline \multirow{3}{*}{0.5} & 0.90 & 29.6030 & 10.4164 & 30.3499 & 10.0334 & 0.2697 & 8.9672 & 3.3284 & 9.7358 & 3.1490 & 0.2321 \\
\hline & 0.95 & 59.1960 & 20.5059 & 59.9570 & 19.8935 & 0.2588 & 16.3966 & 5.8776 & 17.1731 & 5.6288 & 0.2240 \\
\hline & 0.99 & 296.8833 & 101.5791 & 297.6659 & 99.1856 & 0.2506 & 75.8436 & 26.2998 & 76.6260 & 25.5085 & 0.2177 \\
\hline \multirow{3}{*}{0.7} & 0.90 & 29.6030 & 10.4164 & 30.3499 & 10.0334 & 0.2697 & 15.7651 & 5.6915 & 16.5222 & 5.4306 & 0.2438 \\
\hline & 0.95 & 59.1960 & 20.5059 & 59.9570 & 19.8935 & 0.2588 & 30.3062 & 10.6853 & 31.0719 & 10.2887 & 0.2348 \\
\hline & 0.99 & 296.8833 & 101.5791 & 297.6659 & 99.1856 & 0.2506 & 146.8074 & 50.7102 & 147.5796 & 49.2516 & 0.2280 \\
\hline \multirow{3}{*}{0.9} & 0.90 & 29.6030 & 10.4164 & 30.3499 & 10.0334 & 0.2697 & 24.5049 & 8.7316 & 25.2461 & 8.3652 & 0.2600 \\
\hline & 0.95 & 59.1960 & 20.5059 & 59.9570 & 19.8935 & 0.2588 & 48.4960 & 16.9745 & 49.2470 & 16.3840 & 0.2498 \\
\hline & 0.99 & 296.8833 & 101.5791 & 297.6659 & 99.1856 & 0.2506 & 241.0406 & 83.1267 & 241.7988 & 80.7817 & 0.2421 \\
\hline
\end{tabular}


Table C6: EMSEs of estimators for GLMs with gamma response when $\mathrm{n}=600$.

\begin{tabular}{|c|c|c|c|c|c|c|c|c|c|c|c|}
\hline \multirow[b]{3}{*}{ d } & \multirow[b]{3}{*}{ corr } & \multirow[t]{3}{*}{ FOAML } & \multicolumn{4}{|c|}{ FOARML } & \multirow[t]{3}{*}{ FOAL } & \multicolumn{4}{|c|}{ FOARL } \\
\hline & & & & Restric & ions & & & & Restric & tions & \\
\hline & & & (i) & (ii) & (iii) & (iv) & & (i) & (ii) & (iii) & (iv) \\
\hline \multirow{3}{*}{0.1} & 0.90 & 31.0492 & 9.4549 & 31.7829 & 9.7342 & 0.2644 & 1.2202 & 0.6271 & 2.0056 & 0.5519 & 0.2145 \\
\hline & 0.95 & 61.6927 & 18.4864 & 62.4344 & 19.1613 & 0.2527 & 1.4518 & 0.6873 & 2.2449 & 0.6155 & 0.2068 \\
\hline & 0.99 & 306.5041 & 90.7966 & 307.2401 & 94.5599 & 0.2433 & 3.8274 & 1.3867 & 4.6269 & 1.3474 & 0.2005 \\
\hline \multirow{3}{*}{0.3} & 0.90 & 31.0 & 9.4549 & 31.7829 & 9.7342 & 0.2644 & 4.2684 & 1.5579 & 5.0504 & 1.5158 & 0.2178 \\
\hline & 0.95 & 61.6927 & 18.4864 & 62.4344 & 19.1613 & 0.2527 & 7.0191 & 2.3710 & 7.8092 & 2.3675 & 0.2097 \\
\hline & 0.99 & 306.5041 & 90.7966 & 307.2401 & 94.5599 & 0.2433 & 29.0418 & 8.9219 & 29.8386 & 9.2231 & 0.2031 \\
\hline \multirow{3}{*}{0.5} & 0.90 & 31.0492 & 9.4549 & 31.7829 & 9.7342 & 0.2644 & 9.3625 & 3.0874 & 10.1367 & 3.1082 & 0.2255 \\
\hline & 0.95 & 61.6927 & 18.4864 & 62.4344 & 19.1613 & 0.2527 & 17.0548 & 5.3713 & 17.8377 & 5.5007 & 0.2168 \\
\hline & 0.99 & 306.5041 & 90.7966 & 307.2401 & 94.5599 & 0.2433 & 78.2831 & 23.5922 & 79.0733 & 24.5711 & 0.2097 \\
\hline \multirow{3}{*}{0.7} & 0.90 & 31.0492 & 9.4549 & 31.7829 & 9.7342 & 0.2644 & 16.5027 & 5.2156 & 17.2645 & 5.3291 & 0.2377 \\
\hline & 0.95 & 61.6927 & 18.4864 & 62.4344 & 19.1613 & 0.2527 & 31.5587 & 9.6885 & 32.3303 & 10.0153 & 0.2281 \\
\hline & 0.99 & 306.5041 & 90.7966 & 307.2401 & 94.5599 & 0.2433 & 151.5513 & 45.3974 & 152.3309 & 47.3913 & 0.2202 \\
\hline \multirow{3}{*}{0.9} & 0.90 & 31.0492 & 9.4549 & 31.7829 & 9.7342 & 0.2644 & 25.6889 & 7.9426 & 26.4337 & 8.1786 & 0.2544 \\
\hline & 0.95 & 61.6927 & 18.4864 & 62.4344 & 19.1613 & 0.2527 & 50.5310 & 15.3225 & 51.2870 & 15.9110 & 0.2435 \\
\hline & 0.99 & 306.5041 & 90.7966 & 307.2401 & 94.5599 & 0.2433 & 248.8465 & 74.3378 & 249.6116 & 77.6837 & 0.2346 \\
\hline
\end{tabular}

Table C7: EMSEs of estimators for GLMs with gamma response when $\mathrm{n}=700$.

\begin{tabular}{|c|c|c|c|c|c|c|c|c|c|c|c|}
\hline \multirow[b]{3}{*}{$\mathrm{d}$} & \multirow[b]{3}{*}{ corr } & \multirow[t]{3}{*}{ FOAML } & \multirow{2}{*}{\multicolumn{4}{|c|}{ FOARML }} & \multirow[t]{3}{*}{ FOAL } & \multicolumn{4}{|c|}{ FOARL } \\
\hline & & & & & & & & & Restric & tions & \\
\hline & & & (i) & (ii) & (iii) & (iv) & & (i) & (ii) & (iii) & (iv) \\
\hline \multirow{3}{*}{0.1} & 0.90 & 6508 & 10.6061 & 31.4007 & 10.6001 & 0.2682 & 1.2114 & 0.6376 & 1.9914 & 0.5519 & 0.2202 \\
\hline & 0.95 & 61.1418 & 20.8514 & 61.9077 & 20.9660 & 0.2581 & 1.4429 & 0.7122 & 2.2298 & 0.6270 & 0.2132 \\
\hline & 0.99 & 305.5251 & 103.0444 & 306.3232 & 104.1052 & 0.2507 & 3.8167 & 1.5126 & 4.6087 & 1.4331 & 0.2080 \\
\hline \multirow{3}{*}{0.3} & 0.9 & & 1 & 3 & 10.6 & 0.2 & & 1.66 & & & 0.2232 \\
\hline & 0.95 & & 20.8 & 61.9077 & 20.9660 & 0.25 & & 2.5850 & 7.7416 & 2.5029 & 0.2160 \\
\hline & 0.99 & 305.5251 & 103.0444 & 306.3232 & 104.1052 & 0.2507 & 28.9423 & 10.0209 & 29.7317 & 9.9892 & 0.2107 \\
\hline \multirow{3}{*}{0.5} & 0.90 & & & 3 & 10.6 & 0.26 & & 3.3 & & & 0.2305 \\
\hline & 0.95 & 61.1 & 20.8514 & 61.9077 & 20.9660 & 0.2581 & 16.9015 & 5.9606 & 17.6790 & 5.8935 & 0.2229 \\
\hline & 0.99 & 305.5251 & 103.0444 & 306.3232 & 104.1052 & 0.2507 & 78.0226 & 26.6400 & 78.8055 & 26.7119 & 0.2173 \\
\hline \multirow{3}{*}{0.7} & 0.90 & & & & 10.6 & 0.26 & & & 17.0 & & 0.2423 \\
\hline & 0.95 & 61.14 & 20.8514 & 61.9077 & 20.9660 & 0.2581 & 31.2753 & 10.8391 & 32.0420 & 10.7987 & 0.2339 \\
\hline & 0.99 & 305.5251 & 103.0444 & 306.3232 & 104.1052 & 0.2507 & 151.0576 & 51.3701 & 151.8301 & 51.6011 & 0.2277 \\
\hline \multirow{3}{*}{0.9} & 0.90 & & 10.6 & 31.40 & 10.6 & 0.2682 & & 8.8647 & 26.1033 & 8.8041 & 0.2585 \\
\hline & 0.95 & 61.1418 & 20.8514 & 61.9077 & 20.9660 & 0.2581 & 50.0788 & 17.2204 & 50.8307 & 17.2186 & 0.2490 \\
\hline & 0.99 & 305.5251 & 103.0444 & 306.3232 & 104.1052 & 0.2507 & 248.0473 & 84.2112 & 248.8056 & 84.6569 & 0.2421 \\
\hline
\end{tabular}


Table C8: EMSEs of estimators for GLMs with gamma response when $\mathrm{n}=800$.

\begin{tabular}{|c|c|c|c|c|c|c|c|c|c|c|c|}
\hline \multirow[b]{3}{*}{ d } & \multirow[b]{3}{*}{ corr } & \multirow[t]{3}{*}{ FOAML } & \multirow{2}{*}{\multicolumn{4}{|c|}{$\begin{array}{l}\text { FOARML } \\
\text { Restrictions }\end{array}$}} & \multirow[t]{3}{*}{ FOAL } & \multirow{2}{*}{\multicolumn{4}{|c|}{ FOARL }} \\
\hline & & & & & & & & & & & \\
\hline & & & (i) & (ii) & (iii) & (iv) & & (i) & (ii) & (iii) & (iv) \\
\hline \multirow{3}{*}{0.1} & 0.90 & 28.7676 & 9.2882 & 29.4890 & 8.9809 & 0.2787 & 1.1544 & 0.6029 & 1.9432 & 0.5108 & 0.2112 \\
\hline & 0.95 & 57.2782 & 18.1590 & 58.0090 & 17.6806 & 0.2683 & 1.3691 & 0.6659 & 2.1643 & 0.5704 & 0.2048 \\
\hline & 0.99 & 285.5347 & 89.2477 & 286.2683 & 87.3721 & 0.2607 & 3.5840 & 1.3566 & 4.3840 & 1.2416 & 0.2000 \\
\hline \multirow{3}{*}{0.3} & 0.90 & 28.7676 & 9.2882 & 29.4890 & 8.9809 & 0.2787 & 3.9868 & 1.5065 & 4.7690 & 1.3868 & 0.2177 \\
\hline & 0.95 & 57.2782 & 18.1590 & 58.0090 & 17.6806 & 0.2683 & 6.5468 & 2.3050 & 7.3357 & 2.1641 & 0.2111 \\
\hline & 0.99 & 285.5347 & 89.2477 & 286.2683 & 87.3721 & 0.2607 & 27.0810 & 8.7428 & 27.8750 & 8.4313 & 0.2060 \\
\hline \multirow{3}{*}{0.5} & 0.90 & 28.7676 & 9.2882 & 29.4890 & 8.9809 & 0.2787 & 8.7072 & 3.0032 & 9.4780 & 2.8377 & 0.2292 \\
\hline & 0.95 & 57.2782 & 18.1590 & 58.0090 & 17.6806 & 0.2683 & 15.8654 & 5.2434 & 16.6435 & 5.0196 & 0.2218 \\
\hline & 0.99 & 285.5347 & 89.2477 & 286.2683 & 87.3721 & 0.2607 & 72.9545 & 23.1494 & 73.7381 & 22.4508 & 0.2163 \\
\hline \multirow{3}{*}{0.7} & 0.90 & 28.7676 & 9.2882 & 29.4890 & 8.9809 & 0.2787 & 15.3154 & 5.0931 & 16.0700 & 4.8634 & 0.2454 \\
\hline & 0.95 & 57.2782 & 18.1590 & 58.0090 & 17.6806 & 0.2683 & 29.3249 & 9.4810 & 30.0877 & 9.1370 & 0.2370 \\
\hline & 0.99 & 285.5347 & 89.2477 & 286.2683 & 87.3721 & 0.2607 & 141.2043 & 44.5766 & 141.9734 & 43.3001 & 0.2309 \\
\hline \multirow{3}{*}{0.9} & 0.90 & 28.7676 & 9.2882 & 29.4890 & 8.9809 & 0.2787 & 23.8116 & 7.7763 & 24.5451 & 7.4638 & 0.2664 \\
\hline & 0.95 & 57.2782 & 18.1590 & 58.0090 & 17.6806 & 0.2683 & 46.9252 & 15.0178 & 47.6683 & 14.5163 & 0.2568 \\
\hline & 0.99 & 285.5347 & 89.2477 & 286.2683 & 87.3721 & 0.2607 & 231.8305 & 73.0242 & 232.5807 & 70.9791 & 0.2497 \\
\hline
\end{tabular}

Table C9: EMSEs of estimators for GLMs with gamma response when $\mathrm{n}=900$.

\begin{tabular}{|c|c|c|c|c|c|c|c|c|c|c|c|}
\hline \multirow[b]{3}{*}{$\mathrm{d}$} & \multirow[b]{3}{*}{ corr } & \multirow[t]{3}{*}{ FOAML } & \multirow{2}{*}{\multicolumn{4}{|c|}{$\begin{array}{l}\text { FOARML } \\
\text { Restrictions }\end{array}$}} & \multirow[t]{3}{*}{ FOAL } & \multicolumn{4}{|c|}{ FOARL } \\
\hline & & & & & & & & & Restric & ions & \\
\hline & & & (i) & (ii) & (iii) & (iv) & & (i) & (ii) & (iii) & (iv) \\
\hline \multirow{3}{*}{0.1} & 0.90 & 30.8776 & 10.1850 & 31.6047 & 10.4930 & 0.2745 & 1.2322 & 0.6455 & 2.0123 & 0.5616 & 0.2199 \\
\hline & 0.95 & 61.5356 & 19.9866 & 62.2734 & 20.7300 & 0.2637 & 1.4632 & 0.7147 & 2.2502 & 0.6343 & 0.2130 \\
\hline & 0.99 & 307.0856 & 98.6128 & 307.8316 & 102.7954 & 0.2557 & 3.8459 & 1.4826 & 4.6382 & 1.4311 & 0.2078 \\
\hline \multirow{3}{*}{0.3} & 0.90 & 30.8776 & 10.1850 & 31.6047 & 10.4930 & 0.2745 & 4.2710 & 1.6455 & 5.0469 & 1.5851 & 0.2241 \\
\hline & 0.95 & 61.5356 & 19.9866 & 62.2734 & 20.7300 & 0.2637 & 7.0238 & 2.5329 & 7.8069 & 2.5050 & 0.2169 \\
\hline & 0.99 & 307.0856 & 98.6128 & 307.8316 & 102.7954 & 0.2557 & 29.1138 & 9.6898 & 29.9023 & 9.9218 & 0.2114 \\
\hline \multirow{3}{*}{0.5} & 0.90 & 30.8776 & 10.1850 & 31.6047 & 10.4930 & 0.2745 & 9.3379 & 3.2999 & 10.1050 & 3.2911 & 0.2328 \\
\hline & 0.95 & 61.5356 & 19.9866 & 62.2734 & 20.7300 & 0.2637 & 17.0352 & 5.7889 & 17.8102 & 5.8715 & 0.2250 \\
\hline & 0.99 & 307.0856 & 98.6128 & 307.8316 & 102.7954 & 0.2557 & 78.4495 & 25.6918 & 79.2304 & 26.4979 & 0.2191 \\
\hline \multirow{3}{*}{0.7} & 0.90 & 30.8776 & 10.1850 & 31.6047 & 10.4930 & 0.2745 & 16.4327 & 5.6087 & 17.1865 & 5.6796 & 0.2461 \\
\hline & 0.95 & 61.5356 & 19.9866 & 62.2734 & 20.7300 & 0.2637 & 31.4973 & 10.4830 & 32.2599 & 10.7339 & 0.2373 \\
\hline & 0.99 & 307.0856 & 98.6128 & 307.8316 & 102.7954 & 0.2557 & 151.8530 & 49.4887 & 152.6223 & 51.1593 & 0.2308 \\
\hline \multirow{3}{*}{0.9} & 0.90 & 30.8776 & 10.1850 & 31.6047 & 10.4930 & 0.2745 & 25.5556 & 8.5719 & 26.2915 & 8.7505 & 0.2639 \\
\hline & 0.95 & 61.5356 & 19.9866 & 62.2734 & 20.7300 & 0.2637 & 50.4101 & 16.6150 & 51.1562 & 17.0921 & 0.2539 \\
\hline & 0.99 & 307.0856 & 98.6128 & 307.8316 & 102.7954 & 0.2557 & 249.3244 & 81.0805 & 250.0780 & 83.9060 & 0.2464 \\
\hline
\end{tabular}


Table C10: EMSEs of estimators for GLMs with gamma response when $\mathrm{n}=1000$.

\begin{tabular}{|c|c|c|c|c|c|c|c|c|c|c|c|}
\hline \multirow[b]{3}{*}{ d } & \multirow[b]{3}{*}{ corr } & FOAML & \multicolumn{4}{|c|}{ FOARML } & \multirow[t]{3}{*}{ FOAL } & \multicolumn{4}{|c|}{ FOARL } \\
\hline & & & \multicolumn{4}{|c|}{ Restrictions } & & \multicolumn{4}{|c|}{ Restrictions } \\
\hline & & & (i) & (ii) & (iii) & (iv) & & (i) & (ii) & (iii) & (iv) \\
\hline \multirow{3}{*}{0.1} & 0.90 & 29.0214 & 9.3668 & 29.7541 & 9.2088 & 0.2720 & 1.1730 & 0.6068 & 1.9594 & 0.5176 & 0.2137 \\
\hline & 0.95 & 57.9086 & 18.3709 & 58.6554 & 18.1790 & 0.2600 & 1.3884 & 0.6688 & 2.1824 & 0.5785 & 0.2060 \\
\hline & 0.99 & 289.5640 & 90.6743 & 290.3296 & 90.1641 & 0.2506 & 3.6342 & 1.3676 & 4.4345 & 1.2717 & 0.1998 \\
\hline \multirow{3}{*}{0.3} & 0.90 & 29.0 & 9.3668 & 29.7541 & 9.2088 & 0.2720 & 4.03 & 1.5161 & 4.8154 & 1.4136 & 0.2185 \\
\hline & 0.95 & 57.9086 & 18.3709 & 58.6554 & 18.1790 & 0.2600 & 6.6265 & 2.3231 & 7.4161 & 2.2142 & 0.2105 \\
\hline & 0.99 & 289.5640 & 90.6743 & 290.3296 & 90.1641 & 0.2506 & 27.4651 & 8.8525 & 28.2612 & 8.6892 & 0.2039 \\
\hline \multirow{3}{*}{0.5} & 0.90 & 29.0 & 9.3668 & 29.7541 & 9.2088 & 0.2720 & $8.7 \mathrm{~s}$ & 3.0220 & 9.5684 & 2.9015 & 0.2280 \\
\hline & 0.95 & 57.9086 & 18.3709 & 58.6554 & 18.1790 & 0.2600 & 16.0485 & 5.2890 & 16.8294 & 5.1510 & 0.2193 \\
\hline & 0.99 & 289.5640 & 90.6743 & 290.3296 & 90.1641 & 0.2506 & 73.9868 & 23.4544 & 74.7747 & 23.1618 & 0.2122 \\
\hline \multirow{3}{*}{0.7} & 0.90 & 29.0214 & 9.3668 & 29.7541 & 9.208 & 0.2720 & 15.4 & 5.124 & 16.2183 & 4.9815 & 0.2421 \\
\hline & 0.95 & 57.9086 & 18.3709 & 58.6554 & 18.1790 & 0.2600 & 29.6545 & 9.5664 & 30.4224 & 9.3889 & 0.2323 \\
\hline & 0.99 & 289.5640 & 90.6743 & 290.3296 & 90.1641 & 0.2506 & 143.1995 & 45.1733 & 143.9751 & 44.6895 & 0.2245 \\
\hline \multirow{3}{*}{0.9} & 0.90 & 29.0 & 9.3668 & 29.7541 & 9.208 & 0.2720 & 24.0 & 7.8236 & 24.7651 & 7.6534 & 0.2609 \\
\hline & 0.95 & 57.9086 & 18.3709 & 58.6554 & 18.1790 & 0.2600 & 47.4446 & 15.1552 & 48.1952 & 14.9279 & 0.2497 \\
\hline & 0.99 & 289.5640 & 90.6743 & 290.3296 & 90.1641 & 0.2506 & 235.1031 & 74.0092 & 235.8623 & 73.2722 & 0.2409 \\
\hline
\end{tabular}

Table C11: EMSEs of estimators for GLMs with Poisson response when $\mathrm{n}=100$.

\begin{tabular}{|c|c|c|c|c|c|c|c|c|c|c|c|}
\hline \multirow[b]{3}{*}{$\mathrm{d}$} & \multirow[b]{3}{*}{ corr } & \multirow[t]{3}{*}{ FOAML } & \multirow{2}{*}{\multicolumn{4}{|c|}{$\begin{array}{l}\text { FOARML } \\
\text { Restrictions }\end{array}$}} & \multirow[t]{3}{*}{ FOAL } & \multicolumn{4}{|c|}{ FOARL } \\
\hline & & & & & & & & & Restric & tions & \\
\hline & & & (i) & (ii) & (iii) & (iv) & & (i) & (ii) & (iii) & (iv) \\
\hline \multirow{3}{*}{0.1} & 0.90 & 28.5934 & 11.1609 & 31.2301 & 11.4054 & 0.2844 & 1.2374 & 0.6653 & 2.0402 & 0.5681 & 0.2295 \\
\hline & 0.95 & 57.4946 & 21.9826 & 62.2156 & 22.7807 & 0.2741 & 1.4450 & 0.7474 & 2.2483 & 0.6511 & 0.2236 \\
\hline & 0.99 & 290.8867 & 108.3680 & 312.8578 & 114.2393 & 0.2661 & 3.6979 & 1.5955 & 4.5010 & 1.5155 & 0.2180 \\
\hline \multirow{3}{*}{0.3} & 0.90 & 28.5934 & 11.1609 & 31.2301 & 11.4054 & 0.2844 & 4.0791 & 1.7422 & 4.9075 & 1.6391 & 0.2344 \\
\hline & 0.95 & 57.4946 & 21.9826 & 62.2156 & 22.7807 & 0.2741 & 6.6750 & 2.7243 & 7.5087 & 2.6312 & 0.2281 \\
\hline & 0.99 & 290.8867 & 108.3680 & 312.8578 & 114.2393 & 0.2661 & 27.6698 & 10.5985 & 28.5078 & 10.6483 & 0.2224 \\
\hline \multirow{3}{*}{0.5} & 0.90 & 934 & 11.1609 & 31.23 & 11.4054 & 0.28 & & 3.5469 & 9.6233 & 3.4438 & 0.2431 \\
\hline & 0.95 & 57.4946 & 21.9826 & 62.2156 & 22.7807 & 0.2741 & 16.0338 & 6.2959 & 16.8969 & 6.2235 & 0.2362 \\
\hline & 0.99 & 290.8867 & 108.3680 & 312.8578 & 114.2393 & 0.2661 & 74.4118 & 28.1940 & 75.2842 & 28.5201 & 0.2301 \\
\hline \multirow{3}{*}{0.7} & 0.90 & 28.5934 & & 31.2301 & 11.4054 & 0.2844 & 15.3123 & b.0792 & 16.1877 & 5.9822 & 0.2558 \\
\hline & 0.95 & 57.4946 & 21.9826 & 62.2156 & 22.7807 & 0.2741 & 29.5215 & 11.4622 & 30.4131 & 11.4278 & 0.2479 \\
\hline & 0.99 & 290.8867 & 108.3680 & 312.8578 & 114.2393 & 0.2661 & 143.9241 & 54.3819 & 144.8302 & 55.1308 & 0.2412 \\
\hline \multirow{3}{*}{0.9} & 0.90 & 28.5934 & 11.1609 & 31.2301 & 11.4054 & 0.2844 & 23.7039 & 9.3393 & 24.6007 & 9.2544 & 0.2723 \\
\hline & 0.95 & 57.4946 & 21.9826 & 62.2156 & 22.7807 & 0.2741 & 47.1380 & 18.2231 & 48.0572 & 18.2443 & 0.2631 \\
\hline & 0.99 & 290.8867 & 108.3680 & 312.8578 & 114.2393 & 0.2661 & 236.2066 & 89.1623 & 237.1457 & 90.4804 & 0.2557 \\
\hline
\end{tabular}


Table C12: EMSEs of estimators for GLMs with Poisson response when $\mathrm{n}=200$.

\begin{tabular}{|c|c|c|c|c|c|c|c|c|c|c|c|}
\hline \multirow[b]{3}{*}{$\mathrm{d}$} & \multirow[b]{3}{*}{ corr } & \multirow[t]{3}{*}{ FOAML } & \multicolumn{4}{|c|}{ FOARML } & \multirow[t]{3}{*}{ FOAL } & \multicolumn{4}{|c|}{ FOARL } \\
\hline & & & \multicolumn{4}{|c|}{ Restrictions } & & \multicolumn{4}{|c|}{ Restrictions } \\
\hline & & & (i) & (ii) & (iii) & (iv) & & (i) & (ii) & (iii) & (iv) \\
\hline \multirow{3}{*}{0.1} & 0.90 & 30.7105 & 12.2138 & 32.2011 & 11.8859 & 0.2752 & 1. & 0.6582 & 2.0225 & 0.5619 & 0.2139 \\
\hline & 0.95 & 60.7 & 24.0159 & 63.0798 & 23.4800 & 0.2640 & & 0.7482 & 2.2511 & 0.6499 & 0.2077 \\
\hline & 0.99 & 301.0278 & 118.7868 & 310.0946 & 116.2995 & 0.2526 & 3.7785 & 1.6578 & 4.5842 & 1.5424 & 0.2015 \\
\hline \multirow{3}{*}{0.3} & 0.90 & 30 & 12.2138 & 32.2011 & 11.8 & 0.27 & 4.2 & 1.8099 & 5.05 & 1.6 & 0.2193 \\
\hline & 0.95 & 60.7 & 24.0159 & 63.0798 & 23.4800 & 0.2640 & & 2.8543 & 7.7539 & 2.7156 & 0.2126 \\
\hline & 0.99 & 301.0278 & 118.7868 & 310.0946 & 116.2995 & 0.2526 & 28.5607 & 11.2675 & 29.3755 & 10.9749 & 0.2059 \\
\hline \multirow{3}{*}{0.5} & 0.90 & 30 & 12.21 & 32.2011 & 11.8 & 0.27 & 02 & 3.7526 & 10.0940 & 3.5923 & 0.2292 \\
\hline & 0.95 & 60. & 24.0159 & 63.0798 & 23.4800 & 0.2640 & 16.8313 & 6.6741 & 17.6435 & 6.4677 & 0.2217 \\
\hline & 0.99 & 301.0278 & 118.7868 & 310.0946 & 116.2995 & 0.2526 & 76.9276 & 30.0649 & 77.7486 & 29.4308 & 0.2141 \\
\hline \multirow{3}{*}{0.7} & 0.90 & & 12.2138 & 32.2011 & 11.8859 & 0.2752 & & 6.4863 & 17.1481 & 6.2752 & 0.2436 \\
\hline & 0.95 & 60.7527 & 24.0159 & 63.0798 & 23.4800 & 0.2640 & 31.1072 & 12.2077 & 31.9200 & 11.9062 & 0.2349 \\
\hline & 0.99 & 301.0278 & 118.7868 & 310.0946 & 116.2995 & 0.2526 & 148.8792 & 58.0501 & 149.7035 & 56.9103 & 0.2261 \\
\hline \multirow{3}{*}{0.9} & 0.90 & & 12.2138 & 32.2011 & 11.8859 & 0.2752 & 25.4185 & 10.0110 & 26.2145 & 9.7366 & 0.2624 \\
\hline & 0.95 & 60.75 & 24.0159 & 63.0798 & 23.4800 & 0.2640 & 49.7733 & 19.4551 & 50.5834 & 19.0312 & 0.2523 \\
\hline & 0.99 & 301.0278 & 118.7868 & 310.0946 & 116.2995 & 0.2526 & 244.4154 & 95.2229 & 245.2401 & 93.4134 & 0.2419 \\
\hline
\end{tabular}

Table C13: EMSEs of estimators for GLMs with Poisson response when $\mathrm{n}=300$.

\begin{tabular}{|c|c|c|c|c|c|c|c|c|c|c|c|}
\hline \multirow[b]{3}{*}{ d } & \multirow[b]{3}{*}{ corr } & \multirow[t]{3}{*}{ FOAML } & \multirow{2}{*}{\multicolumn{4}{|c|}{ FOARML }} & \multirow[t]{3}{*}{ FOAL } & \multicolumn{4}{|c|}{ FOARL } \\
\hline & & & & & & & & & Restr & tions & \\
\hline & & & (i) & (ii) & (iii) & (iv) & & (i) & (ii) & (iii) & (iv) \\
\hline \multirow{3}{*}{0.1} & 0.90 & 6153 & 10.0287 & 31.7611 & 10.0938 & 0.2783 & 1.2322 & 0.6549 & 2.0277 & 0.5615 & 0.2240 \\
\hline & 0.95 & 59.3247 & 19.6938 & 62.9979 & 19.9774 & 0.2664 & & 0.7180 & 2.2499 & 0.6266 & 0.2162 \\
\hline & 0.99 & 297.9529 & 97.6277 & 313.8923 & 99.6760 & 0.2581 & 3.7596 & 1.4641 & 4.5615 & 1.3865 & 0.2112 \\
\hline \multirow{3}{*}{0.3} & 0.90 & 3 & 10.0 & 31.76 & 10.0 & 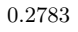 & & 9 & 4.9763 & 1.5379 & 0.2285 \\
\hline & 0.95 & 59.3 & 19.6938 & 62.9979 & 19.9774 & 0.2664 & 6.8319 & 2.4889 & 7.6509 & 2.4094 & 0.2203 \\
\hline & 0.99 & 297.9529 & 97.6277 & 313.8923 & 99.6760 & 0.2581 & 28.2971 & 9.4620 & 29.1206 & 9.5031 & 0.2149 \\
\hline \multirow{3}{*}{0.5} & 0.90 & & 1 & 31.76 & 10.0938 & 0 . & & 3.2 & 9.8511 & 3.1538 & 0.2372 \\
\hline & 0.95 & 47 & 19.6938 & 62.9979 & 19.9774 & 0.2664 & 16.4877 & 5.6463 & 17.3237 & 5.6044 & 0.2283 \\
\hline & 0.99 & 297.9529 & 97.6277 & 313.8923 & 99.6760 & 0.2581 & 76.1710 & 25.0394 & 77.0148 & 25.3345 & 0.2224 \\
\hline \multirow{3}{*}{0.7} & 0.90 & & 1 & 31.7 & 10.0 & 0 . & & 5.4 & 16.6 & 5.4 & 0.2501 \\
\hline & 0.95 & 59.3 & 19 & 62.99 & 19.9774 & 0.2 & 30. & 10.1 & 31.2685 & 10.2115 & 0.2402 \\
\hline & 0.99 & 297.9529 & 97.6277 & 313.8923 & 99.6760 & 0.2581 & 147.3814 & 48.1965 & 148.2440 & 48.8806 & 0.2335 \\
\hline \multirow{3}{*}{0.9} & 0.90 & & 10. & 31.76 & & 0.2 & & 8.32 & 25.3 & & 0.2671 \\
\hline & 0.95 & 59.3247 & 19.6938 & 62.9979 & 19.9774 & 0.2664 & 48.6204 & 16.1202 & 49.4851 & 16.2308 & 0.2560 \\
\hline & 0.99 & 297.9529 & 97.6277 & 313.8923 & 99.6760 & 0.2581 & 241.9283 & 78.9330 & 242.8081 & 80.1415 & 0.2484 \\
\hline
\end{tabular}


Table C14: EMSEs of estimators for GLMs with Poisson response when $\mathrm{n}=400$.

\begin{tabular}{|c|c|c|c|c|c|c|c|c|c|c|c|}
\hline \multirow[b]{3}{*}{ d } & \multirow[b]{3}{*}{ corr } & \multirow[t]{3}{*}{ FOAML } & \multicolumn{4}{|c|}{ FOARML } & \multirow[t]{3}{*}{ FOAL } & \multicolumn{4}{|c|}{ FOARL } \\
\hline & & & & Restric & ions & & & & Restric & tions & \\
\hline & & & (i) & (ii) & (iii) & (iv) & & (i) & (ii) & (iii) & (iv) \\
\hline \multirow{3}{*}{0.1} & 0.90 & 30.7848 & 10.1645 & 32.3040 & 10.0751 & 0.2847 & 1.2492 & 0.6552 & 2.0313 & 0.5654 & 0.2292 \\
\hline & 0.95 & 61.3707 & 19.9339 & 63.7764 & 19.8870 & 0.2734 & 1.4794 & 0.7232 & 2.2669 & 0.6329 & 0.2222 \\
\hline & 0.99 & 305.0298 & 97.5975 & 314.5162 & 97.9331 & 0.2663 & 3.8416 & 1.4750 & 4.6314 & 1.3826 & 0.2181 \\
\hline \multirow{3}{*}{0.3} & 0.90 & 30.7 & 10.1645 & 32.3040 & 10.0751 & 0.2847 & 4.2842 & 1.6473 & 5.0739 & 1.5452 & 0.2338 \\
\hline & 0.95 & 61.3707 & 19.9339 & 63.7764 & 19.8870 & 0.2734 & 7.0330 & 2.5256 & 7.8301 & 2.4207 & 0.2264 \\
\hline & 0.99 & 305.0298 & 97.5975 & 314.5162 & 97.9331 & 0.2663 & 28.9513 & 9.5400 & 29.7526 & 9.4166 & 0.2221 \\
\hline \multirow{3}{*}{0.5} & 0.90 & 30.7848 & 10.1645 & 32.3040 & 10.0751 & 0.2847 & 9.3355 & 3.2860 & 10.1296 & 3.1692 & 0.2427 \\
\hline & 0.95 & 61.3707 & 19.9339 & 63.7764 & 19.8870 & 0.2734 & 17.0183 & 5.7497 & 17.8224 & 5.6255 & 0.2346 \\
\hline & 0.99 & 305.0298 & 97.5975 & 314.5162 & 97.9331 & 0.2663 & 77.9587 & 25.2596 & 78.7692 & 25.0840 & 0.2298 \\
\hline \multirow{3}{*}{0.7} & 0.90 & 30.7848 & 10.1645 & 32.3040 & 10.0751 & 0.2847 & 16.4030 & 5.5711 & 17.1985 & 5.4374 & 0.2559 \\
\hline & 0.95 & 61.3707 & 19.9339 & 63.7764 & 19.8870 & 0.2734 & 31.4355 & 10.3957 & 32.2439 & 10.2473 & 0.2468 \\
\hline & 0.99 & 305.0298 & 97.5975 & 314.5162 & 97.9331 & 0.2663 & 150.8639 & 48.6337 & 151.6813 & 48.3848 & 0.2413 \\
\hline \multirow{3}{*}{0.9} & 0.90 & 30.7848 & 10.1645 & 32.3040 & 10.0751 & 0.2847 & 25.4868 & 8.5028 & 26.2807 & 8.3499 & 0.2734 \\
\hline & 0.95 & 61.3707 & 19.9339 & 63.7764 & 19.8870 & 0.2734 & 50.2843 & 16.4634 & 51.0945 & 16.2863 & 0.2630 \\
\hline & 0.99 & 305.0298 & 97.5975 & 314.5162 & 97.9331 & 0.2663 & 247.6667 & 79.6623 & 248.4888 & 79.3191 & 0.2566 \\
\hline
\end{tabular}

Table C15: EMSEs of estimators for GLMs with Poisson response when $\mathrm{n}=500$.

\begin{tabular}{|c|c|c|c|c|c|c|c|c|c|c|c|}
\hline \multirow[b]{3}{*}{$\mathrm{d}$} & \multirow[b]{3}{*}{ corr } & \multirow[t]{3}{*}{ FOAML } & \multirow{2}{*}{\multicolumn{4}{|c|}{$\begin{array}{l}\text { FOARML } \\
\text { Restrictions }\end{array}$}} & \multirow[t]{3}{*}{ FOAL } & \multicolumn{4}{|c|}{ FOARL } \\
\hline & & & & & & & & & Restric & tions & \\
\hline & & & (i) & (ii) & (iii) & (iv) & & (i) & (ii) & (iii) & (iv) \\
\hline \multirow{3}{*}{0.1} & 0.90 & 29.1453 & 10.4823 & 30.2451 & 10.2481 & 0.2666 & 1.1867 & 0.6286 & 1.9790 & 0.5464 & 0.2130 \\
\hline & 0.95 & 58.3401 & 20.6028 & 59.8329 & 20.2990 & 0.2566 & 1.4050 & 0.7008 & 2.2027 & 0.6162 & 0.2067 \\
\hline & 0.99 & 292.0949 & 101.8702 & 296.6694 & 101.1129 & 0.2499 & 3.6703 & 1.4824 & 4.4718 & 1.3897 & 0.2021 \\
\hline \multirow{3}{*}{0.3} & 0.90 & 29.1453 & 10.4823 & 30.2451 & 10.2481 & 0.26 & 4.06 & 1.6324 & 4.8586 & 1.5351 & 0.2169 \\
\hline & 0.95 & 58.3401 & 20.6028 & 59.8329 & 20.2990 & 0.2566 & 6.6869 & 2.5312 & 7.4875 & 2.4249 & 0.2104 \\
\hline & 0.99 & 292.0949 & 101.8702 & 296.6694 & 101.1129 & 0.2499 & 27.7135 & 9.7979 & 28.5185 & 9.6280 & 0.2057 \\
\hline \multirow{3}{*}{0.5} & 0.90 & 29.1453 & 10.48 & 30.2 & 10.2481 & 0.2666 & & 3.3050 & 9.6401 & 3.1798 & 0.2254 \\
\hline & 0.95 & 58.3401 & 20.6028 & 59.8329 & 20.2990 & 0.2566 & 16.1805 & 5.8267 & 16.9803 & 5.6770 & 0.2183 \\
\hline & 0.99 & 292.0949 & 101.8702 & 296.6694 & 101.1129 & 0.2499 & 74.6430 & 26.0374 & 75.4483 & 25.7097 & 0.2132 \\
\hline \multirow{3}{*}{0.7} & 0.90 & 29.1453 & 10.4823 & 30.2451 & 10.2481 & 0.2666 & 15.5 & 5.6465 & 16.3237 & 5.4806 & 0.2383 \\
\hline & 0.95 & 58.3401 & 20.6028 & 59.8329 & 20.2990 & 0.2566 & 29.8856 & 10.5873 & 30.6812 & 10.3724 & 0.2303 \\
\hline & 0.99 & 292.0949 & 101.8702 & 296.6694 & 101.1129 & 0.2499 & 144.4590 & 50.2010 & 145.2612 & 49.6347 & 0.2247 \\
\hline \multirow{3}{*}{0.9} & 0.90 & 29.1453 & 10.4823 & 30.2451 & 10.2481 & 0.2666 & 24.1329 & 8.6568 & 24.9093 & 8.4374 & 0.2557 \\
\hline & 0.95 & 58.3401 & 20.6028 & 59.8329 & 20.2990 & 0.2566 & 47.8024 & 16.8129 & 48.5902 & 16.5112 & 0.2465 \\
\hline & 0.99 & 292.0949 & 101.8702 & 296.6694 & 101.1129 & 0.2499 & 237.1613 & 82.2886 & 237.9574 & 81.4031 & 0.2402 \\
\hline
\end{tabular}


Table C16: EMSEs of estimators for GLMs with Poisson response when $\mathrm{n}=600$.

\begin{tabular}{|c|c|c|c|c|c|c|c|c|c|c|c|}
\hline \multirow[b]{3}{*}{$\mathrm{d}$} & \multirow[b]{3}{*}{ corr } & \multirow[t]{3}{*}{ FOAML } & \multirow{2}{*}{\multicolumn{4}{|c|}{ FOARML }} & \multirow[t]{3}{*}{ FOAL } & \multicolumn{4}{|c|}{ FOARL } \\
\hline & & & & & & & & & Restric & tions & \\
\hline & & & (i) & (ii) & (iii) & (iv) & & (i) & (ii) & (iii) & (iv) \\
\hline \multirow{3}{*}{0.1} & 0.90 & 2 ? & 9.1261 & 31.2383 & 9.1824 & 0.2779 & & 0.6252 & 1.99 & 0.5368 & 0.2166 \\
\hline & 0.9 & 59.06 & 17.7827 & 61.3714 & 18.0406 & 0.2659 & & 0.68 & 2.21 & 0.5954 & 0.2091 \\
\hline & 0.99 & 293.6343 & 86.8990 & 302.4162 & 88.5403 & 0.2560 & 3.6892 & 1.3474 & 4.4937 & 1.2754 & 0.2026 \\
\hline \multirow{3}{*}{0.3} & & & 9 & 3 & & 0 . & & & 4.9 & & 0.2223 \\
\hline & 0.9 & & 17.78 & 61.3714 & 18.0406 & 0.2 & & 2.3003 & 7.5651 & & 0.2144 \\
\hline & 0.99 & 293.6343 & 86.8990 & 302.4162 & 88.5403 & 0.2560 & 27.8600 & 8.5508 & 28.6733 & 8.6270 & 0.2076 \\
\hline \multirow{3}{*}{0.5} & & & 9 & 31.2 & & & & & & & 0.2325 \\
\hline & 0.95 & 59.0 & 17.7827 & 61.3714 & 18.0406 & 0.2 & 16.3 & 5.1780 & 17.1800 & 5.1781 & 0.2238 \\
\hline & 0.99 & 293.6343 & 86.8990 & 302.4162 & 88.5403 & 0.2560 & 75.0374 & 22.5665 & 75.8570 & 22.9457 & 0.2164 \\
\hline \multirow{3}{*}{0.7} & 0.90 & & 8.1201 & 31.2383 & & & & & 16.6 & 5.0 & 0.2471 \\
\hline & 0.95 & $5 !$ & 17.7827 & 61.3714 & 18.0406 & 0.2659 & 30.2 & 9.3149 & 31.0597 & 9.4064 & 0.2373 \\
\hline & 0.99 & 293.6343 & 86.8990 & 302.4162 & 88.5403 & 0.2560 & 145.2213 & 43.3944 & 146.0445 & 44.2314 & 0.2291 \\
\hline \multirow{3}{*}{0.9} & 0.9 & & 9.1261 & 31.2383 & 9.1824 & 0.2 & & 7.6554 & 25.4200 & 7.6 & 0.2661 \\
\hline & 0.9 & $5 \mathrm{~s}$ & 17.7827 & 61.3714 & 18.0406 & 0.2659 & 48.3 & 14.7109 & 49.2044 & 14.9259 & 0.2549 \\
\hline & 0.99 & 293.6343 & 86.8990 & 302.4162 & 88.5403 & 0.2560 & 238.4117 & 71.0346 & 239.2361 & 72.4843 & 0.2457 \\
\hline
\end{tabular}

Table C17: EMSEs of estimators for GLMs with Poisson response when $\mathrm{n}=700$.

\begin{tabular}{|c|c|c|c|c|c|c|c|c|c|c|c|}
\hline \multirow[b]{3}{*}{ d } & \multirow[b]{3}{*}{ corr } & \multirow[t]{3}{*}{ FOAML } & \multirow{2}{*}{\multicolumn{4}{|c|}{$\begin{array}{l}\text { FOARML } \\
\text { Restrictions }\end{array}$}} & \multirow[t]{3}{*}{ FOAL } & \multicolumn{4}{|c|}{ FOARL } \\
\hline & & & & & & & & & Restric & tions & \\
\hline & & & (i) & (ii) & (iii) & (iv) & & (i) & (ii) & (iii) & (iv) \\
\hline \multirow{3}{*}{0.1} & 0.90 & 30.4922 & 10.1295 & 31.5624 & 10.3847 & 0.2690 & 1.2144 & 0.6259 & 2.0009 & 0.5451 & 0.2183 \\
\hline & 0.95 & 60.7094 & 19.9315 & 62.1660 & 20.5798 & 0.2590 & 1.4416 & 0.6975 & 2.2339 & 0.6194 & 0.2119 \\
\hline & 0.99 & 303.3710 & 97.9897 & 307.8700 & 101.8113 & 0.2507 & 3.7972 & 1.4554 & 4.5940 & 1.4042 & 0.2066 \\
\hline \multirow{3}{*}{0.3} & 0.90 & 30.4922 & 10.1295 & 31.5624 & 10.3847 & 0.2690 & 4.2134 & 1.6067 & 5.0014 & 1.5479 & 0.2219 \\
\hline & 0.95 & 60.7094 & 19.9315 & 62.1660 & 20.5798 & 0.2590 & 6.9246 & 2.4889 & 7.7194 & 2.4602 & 0.2152 \\
\hline & 0.99 & 303.3710 & 97.9897 & 307.8700 & 101.8113 & 0.2507 & 28.7549 & 9.5415 & 29.5553 & 9.7594 & 0.2097 \\
\hline \multirow{3}{*}{0.5} & 0.90 & 30.4922 & 10.1295 & 31.5624 & 10.3847 & 0.2690 & 9.2165 & 3.2409 & 10.0023 & 3.2276 & 0.2299 \\
\hline & 0.95 & 60.7094 & 19.9315 & 62.1660 & 20.5798 & 0.2590 & 16.8005 & 5.7125 & 17.5945 & 5.7842 & 0.2226 \\
\hline & 0.99 & 303.3710 & 97.9897 & 307.8700 & 101.8113 & 0.2507 & 77.4921 & 25.3281 & 78.2930 & 26.0856 & 0.2166 \\
\hline \multirow{3}{*}{0.7} & 0.90 & 30.4922 & 10.1295 & 31.5624 & 10.3847 & 0.2690 & 16.2237 & 5.5283 & 17.0035 & 5.5841 & 0.2422 \\
\hline & 0.95 & 60.7094 & 19.9315 & 62.1660 & 20.5798 & 0.2590 & 31.0694 & 10.3684 & 31.8592 & 10.5912 & 0.2340 \\
\hline & 0.99 & 303.3710 & 97.9897 & 307.8700 & 101.8113 & 0.2507 & 150.0090 & 48.8153 & 150.8072 & 50.3828 & 0.2273 \\
\hline \multirow{3}{*}{0.9} & 0.90 & 30.4922 & 10.1295 & 31.5624 & 10.3847 & 0.2690 & 25.2350 & 8.4690 & 26.0050 & 8.6176 & 0.2587 \\
\hline & 0.95 & 60.7094 & 19.9315 & 62.1660 & 20.5798 & 0.2590 & 49.7312 & 16.4565 & 50.5134 & 16.8814 & 0.2494 \\
\hline & 0.99 & 303.3710 & 97.9897 & 307.8700 & 101.8113 & 0.2507 & 246.3054 & 80.0030 & 247.0979 & 82.6510 & 0.2417 \\
\hline
\end{tabular}


Table C18: EMSEs of estimators for GLMs with Poisson response when $\mathrm{n}=800$.

\begin{tabular}{|c|c|c|c|c|c|c|c|c|c|c|c|}
\hline \multirow[b]{3}{*}{ d } & \multirow[b]{3}{*}{ corr } & \multirow[t]{3}{*}{ FOAML } & \multicolumn{4}{|c|}{ FOARML } & \multirow[t]{3}{*}{ FOAL } & \multicolumn{4}{|c|}{ FOARL } \\
\hline & & & & Restric & ions & & & & Restric & tions & \\
\hline & & & (i) & (ii) & (iii) & (iv) & & (i) & (ii) & (iii) & (iv) \\
\hline \multirow{3}{*}{0.1} & 0.90 & 30.1563 & 10.2279 & 30.9995 & 9.9267 & 0.2666 & 1.2082 & 0.6329 & 1.9987 & 0.5441 & 0.2113 \\
\hline & 0.95 & 60.0867 & 20.0413 & 61.0733 & 19.5458 & 0.2575 & 1.4335 & 0.7025 & 2.2299 & 0.6101 & 0.2052 \\
\hline & 0.99 & 299.6464 & 98.7883 & 301.7316 & 96.7418 & 0.2499 & 3.7574 & 1.4645 & 4.5581 & 1.3524 & 0.2006 \\
\hline \multirow{3}{*}{0.3} & 0.90 & 30 & 10.2279 & 30.9995 & 9.9267 & 0.2666 & 4.18 & 1.6256 & 4.9695 & 1.5113 & 0.2157 \\
\hline & 0.95 & 60.0867 & 20.0413 & 61.0733 & 19.5458 & 0.2575 & 6.8701 & 2.5048 & 7.6645 & 2.3682 & 0.2095 \\
\hline & 0.99 & 299.6464 & 98.7883 & 301.7316 & 96.7418 & 0.2499 & 28.4215 & 9.6027 & 29.2209 & 9.2942 & 0.2046 \\
\hline \multirow{3}{*}{0.5} & 0.90 & 30.1563 & 10.2279 & 30.9995 & 9.9267 & 0.2666 & 9.1316 & 3.2706 & 9.9129 & 3.1131 & 0.2245 \\
\hline & 0.95 & 60.0867 & 20.0413 & 61.0733 & 19.5458 & 0.2575 & 16.6480 & 5.7361 & 17.4366 & 5.5184 & 0.2179 \\
\hline & 0.99 & 299.6464 & 98.7883 & 301.7316 & 96.7418 & 0.2499 & 76.5650 & 25.4768 & 77.3595 & 24.7803 & 0.2125 \\
\hline \multirow{3}{*}{0.7} & 0.90 & 30.1563 & 10.2279 & 30.9995 & 9.9267 & 0.2666 & 16.0588 & 5.5680 & 16.8290 & 5.3495 & 0.2379 \\
\hline & 0.95 & 60.0867 & 20.0413 & 61.0733 & 19.5458 & 0.2575 & 30.7674 & 10.3964 & 31.5461 & 10.0606 & 0.2304 \\
\hline & 0.99 & 299.6464 & 98.7883 & 301.7316 & 96.7418 & 0.2499 & 148.1880 & 49.0869 & 148.9738 & 47.8107 & 0.2244 \\
\hline \multirow{3}{*}{0.9} & 0.90 & 30.1563 & 10.2279 & 30.9995 & 9.9267 & 0.2666 & 24.9629 & 8.5178 & 25.7177 & 8.2207 & 0.2556 \\
\hline & 0.95 & 60.0867 & 20.0413 & 61.0733 & 19.5458 & 0.2575 & 49.2282 & 16.4859 & 49.9931 & 15.9949 & 0.2472 \\
\hline & 0.99 & 299.6464 & 98.7883 & 301.7316 & 96.7418 & 0.2499 & 243.2904 & 80.4329 & 244.0638 & 78.3853 & 0.2402 \\
\hline
\end{tabular}

Table C19: EMSEs of estimators for GLMs with Poisson response when $\mathrm{n}=900$.

\begin{tabular}{|c|c|c|c|c|c|c|c|c|c|c|c|}
\hline \multirow[b]{3}{*}{ d } & \multirow[b]{3}{*}{ corr } & \multirow[t]{3}{*}{ FOAML } & \multirow{2}{*}{\multicolumn{4}{|c|}{$\begin{array}{l}\text { FOARML } \\
\text { Restrictions }\end{array}$}} & \multirow[t]{3}{*}{ FOAL } & \multicolumn{4}{|c|}{ FOARL } \\
\hline & & & & & & & & & Restric & ions & \\
\hline & & & (i) & (ii) & (iii) & (iv) & & (i) & (ii) & (iii) & (iv) \\
\hline \multirow{3}{*}{0.1} & 0.90 & 29.3950 & 9.3950 & 30.4309 & 9.5818 & 0.2676 & 1.1 & 0.6134 & 1.9774 & 0.5261 & 0.2133 \\
\hline & 0.95 & 58.6 & 18.3986 & 60.0636 & 18.8864 & 0.25 & & 0.6754 & 2.2041 & 0.5905 & 0.2062 \\
\hline & 0.99 & 292.5257 & 90.3224 & 296.7109 & 93.5075 & 0.2492 & 3.6737 & 1.3723 & 4.4757 & 1.3122 & 0.2012 \\
\hline \multirow{3}{*}{0.3} & 0. & ) & 0 & 30.4309 & 8 & 0.26 & & 1.5 & 4.8 & & 0.2175 \\
\hline & 0.95 & 58.6 & 18.3986 & 60.0636 & 18.8864 & 0.2570 & & 2.3 & 7.5142 & 2.2896 & 0.2101 \\
\hline & 0.99 & 292.5257 & 90.3224 & 296.7109 & 93.5075 & 0.2492 & 27.7514 & 8.8481 & 28.5559 & 9.0142 & 0.2049 \\
\hline \multirow{3}{*}{0.5} & & & 年 & 30.43 & 9 & 0.2 & & & 9.6960 & & 0.2261 \\
\hline & 0.95 & 58.6 & 18.3986 & 60.0636 & 18.8864 & 0.2570 & & 5.3 & 17.0592 & 5.3449 & 0.2182 \\
\hline & 0.99 & 292.5257 & 90.3224 & 296.7109 & 93.5075 & 0.2492 & 74.7500 & 23.4210 & 75.5537 & 24.0473 & 0.2125 \\
\hline \multirow{3}{*}{0.7} & 0.90 & & & 50.4503 & 5.00 & & & & 16.4387 & 5.1 & 0.2392 \\
\hline & 0.95 & 58 & 18.3986 & 60.0636 & 18.8864 & 0.2 & & 9.6047 & 30.8392 & 9.7564 & 0.2304 \\
\hline & 0.99 & 292.5257 & 90.3224 & 296.7109 & 93.5075 & 0.2492 & 144.6696 & 45.0912 & 145.4692 & 46.4113 & 0.2241 \\
\hline \multirow{3}{*}{0.9} & 0.90 & & & 30.4309 & 9.00 & 0.26 & & 7.86 & 25.1037 & 7.9689 & 0.2568 \\
\hline & 0.95 & & 18.3986 & 60.0636 & 18.8864 & 0.25 & & 15.2065 & 48.8542 & 15.5242 & 0.2468 \\
\hline & 0.99 & 292.5257 & 90.3224 & 296.7109 & 93.5075 & 0.2492 & 237.5101 & 73.8585 & 238.3024 & 76.1064 & 0.2396 \\
\hline
\end{tabular}


Table C20: EMSEs of estimators for GLMs with Poisson response when $\mathrm{n}=1000$.

\begin{tabular}{|c|c|c|c|c|c|c|c|c|c|c|c|}
\hline \multirow[b]{3}{*}{ d } & \multirow[b]{3}{*}{ corr } & \multirow[t]{3}{*}{ FOAML } & \multirow{2}{*}{\multicolumn{4}{|c|}{$\begin{array}{l}\text { FOARML } \\
\text { Restrictions }\end{array}$}} & \multirow[t]{3}{*}{ FOAL } & \multicolumn{4}{|c|}{ FOARL } \\
\hline & & & & & & & & & Restric & ions & \\
\hline & & & (i) & (ii) & (iii) & (iv) & & (i) & (ii) & (iii) & (iv) \\
\hline \multirow{3}{*}{0.1} & 0.90 & 30.0800 & 10.1238 & 31.0495 & 10.1334 & 0.2765 & 1.2220 & 0.6406 & 2.0077 & 0.5543 & 0.2178 \\
\hline & 0.95 & 59.9603 & 19.9813 & 61.2093 & 20.1167 & 0.2667 & 1.4447 & 0.7114 & 2.2360 & 0.6250 & 0.2116 \\
\hline & 0.99 & 299.5451 & 98.5618 & 302.9818 & 99.6335 & 0.2589 & 3.7670 & 1.4701 & 4.5626 & 1.3887 & 0.2068 \\
\hline \multirow{3}{*}{0.3} & 0.90 & 30.0 & 10.1238 & 31.0495 & 10.1334 & 0.2765 & 4.1 & 1.6 & 4.9776 & 1.5408 & 0.2229 \\
\hline & 0.95 & 59.9603 & 19.9813 & 61.2093 & 20.1167 & 0.2667 & 6.8758 & 2.5116 & 7.6666 & 2.4326 & 0.2164 \\
\hline & 0.99 & 299.5451 & 98.5618 & 302.9818 & 99.6335 & 0.2589 & 28.4283 & 9.5852 & 29.2242 & 9.5561 & 0.2114 \\
\hline \multirow{3}{*}{0.5} & 0.90 & 30.0800 & 10.1238 & 31.0495 & 10.1334 & 0.2765 & 9.1 & 3.2556 & 9.9101 & 3.1767 & 0.2324 \\
\hline & 0.95 & 59.9603 & 19.9813 & 61.2093 & 20.1167 & 0.2667 & 16.6339 & 5.7342 & 17.4206 & 5.6747 & 0.2255 \\
\hline & 0.99 & 299.5451 & 98.5618 & 302.9818 & 99.6335 & 0.2589 & 76.5565 & 25.4075 & 77.3493 & 25.4878 & 0.2199 \\
\hline \multirow{3}{*}{0.7} & 0.90 & 30.0800 & 10.1238 & 31.0495 & 10.1334 & 0.2765 & 16.0 & 5.5285 & 16.8051 & 5.4621 & 0.2465 \\
\hline & 0.95 & 59.9603 & 19.9813 & 61.2093 & 20.1167 & 0.2667 & 30.7192 & 10.3795 & 31.4978 & 10.3515 & 0.2387 \\
\hline & 0.99 & 299.5451 & 98.5618 & 302.9818 & 99.6335 & 0.2589 & 148.1518 & 48.9371 & 148.9378 & 49.1836 & 0.2324 \\
\hline \multirow{3}{*}{0.9} & 0.90 & 30.0800 & 10.1238 & 31.0495 & 10.1334 & 0.2765 & 24.9068 & 8.4450 & 25.6628 & 8.3969 & 0.2651 \\
\hline & 0.95 & 59.9603 & 19.9813 & 61.2093 & 20.1167 & 0.2667 & 49.1315 & 16.4472 & 49.8984 & 16.4629 & 0.2561 \\
\hline & 0.99 & 299.5451 & 98.5618 & 302.9818 & 99.6335 & 0.2589 & 243.2139 & 80.1738 & 243.9898 & 80.6436 & 0.2489 \\
\hline
\end{tabular}

\section{References}

[1] D. A. Belsley, E. Kuh and R. E. Welsch. Regression diagnostics:Identifying influential data and sources of collinearity, John Wiley and Sons, 1980.

[2] A. Ben-Israel and T. N. E. Greville. Generalized inverses: Theory and applications, John Wiley and Sons, 1974.

[3] S. Chatterjee and A. S. Hadi. Sensitivity analysis in linear regression, John Wiley and Sons, 1988.

[4] F. A. Graybill. Theory and application of the linear model, Duxbury Press, 1976.

[5] J. W. Hardin and J. M. Hilbe. Generalized linear models and extensions, Stata Press, 2012.

[6] D. A. Harville. Matrix algebra from a statistician's perspective, Springer, 2008.

[7] S. Kaçıranlar, S. Sakallığlu, F. Akdeniz, G. P. Styan and H. J. Werner. A new biased estimator in linear regression and a detailed analysis of the widely-analysed dataset on Portland cement, Sankhya: The Indian J Stat Ser B. 61, 443-459, 1999.

[8] F. Kurtoğlu and M. R. Özkale. Liu estimation in generalized linear models: application on gamma distributed response variable, Statistical Papers 57(4), 911-928, 2016.

[9] K. Liu. A new class of biased estimate in linear regression, Comm Statist Theory Methods 22(2), 393-402, 1993.

[10] M. J. Mackinnon and M. L. Puterman. Collinearity in generalized linear models, Comm Statist Theory Methods 18(9), 3463-3472, 1989.

[11] B. D. Marx. A continuum of principal component generalized linear regressions, Comput Statist Data Analysis 13, 385-393, 1992.

[12] B. D. Marx and E. P. Smith. Principal component estimation for generalized linear regression, Biometrika 77(1), 23-31, 1990.

[13] G. C. McDonald and D. I. Galarneau. A monte carlo evaluation of ridge-type estimators, J Amer Statist Assoc. 70, 407-416, 1975.

[14] R. H. Myers. Classical and modern regression with applications, Duxbury Press, 1990.

[15] H. Nyquist. Restricted estimation of generalized linear models, J Roy Statist Soc Ser C. 40(1), 133-141, 1991.

[16] M. R. Özkale. The relative efficiency of the restricted estimators in linear regression models, J App Statist. 41(5), 998-1027, 2014.

[17] B. Segerstedt. On ordinary ridge regression in generalized linear models, Commun Statist Theory Methods 21(8), 2227-2246, 1992.

[18] G. Trenkler and H. Toutenburg. Mean squared error matrix comparisons between biased estimators an overview of recent results, Statistical Papers 31(1), 165-179, 1990. 